\title{
Dry Markets and Statistical A rbitrage Bounds for European Derivatives
}

\author{
J oao Amaro de Matos \\ Faculdade de E conomia \\ Universidade Nova de Lisboa \\ Campus de Campolide \\ 1099-032 Lisboa, Portugal
}

\author{
Ana Lacerda \\ Department of Statistics \\ Columbia University \\ 1255 A msterdam Avenue \\ New Y ork, NY 100027, USA
}

J anuary 2, 2006

\begin{abstract}
A bstract
We derive statistical arbitrage bounds for the buying and selling price of European derivatives under incomplete markets. In this paper, incompleteness is generated due to the fact that the market is dry, i.e., the underlying asset cannot be transact ed at certain points in time. In particular, we re ${ }^{-}$ne the notion of statistical arbitrage in order to extend the procedure for the case where dryness is random, i.e., at each point in time the asset can be transacted with a given probability. We analytically characterize several properties of the statistical arbitragefree interval, show that it is narrower than the super-replication interval and dominates somehow alternative intervals provided in the literature. Moreover, we show that, for su \pm ciently incomplete markets, the statistical arbitrage interval contains the reservation price of the derivative.
\end{abstract}

\section{Introduction}

In complete markets and under the absence of arbitrage opportunities, the value of a E uropean derivative must be the same as the cheapest portfolio that replicates exactly its value at any given point in time. However, in the presence of some market imperfections, markets may become incomplete, and it is not possibleto exactly replicate the value of theE uropean derivative at all times anymore. Nevertheless, it is possible to derive an arbitrage free range of variation for the value of the derivative. This interval depends on 
two di ßerent factors. F irst, on the nature of market incompleteness; second, on the notion of arbitrage opportunities.

In what follows we consider that market incompleteness is generated by the fact that agents cannot trade the underlying asset on which the derivative is written whenever they please. In fact, and as opposed to the traditional asset pricing assumptions, markets are very rarely liquid and immediacy is not always available. As Longsta ${ }^{(1995,2001,2004)}$ recalls, the relevance of this fact is pervasive through many " nancial markets. The markets for many assets such as human capital, business partnerships, pensi on plans, saving bonds, annuities, trusts, inheritances and residential real estate, among others, are generally very illiquid and long periods of time (months, sometimes years) may be required to sell an asset. This point becomes extremely relevant for the case of option pricing when we consider that it is an increasingly common phenomenon even in well-established securities markets, as illustrated by the 1998 Russian default crisis, leading many traders to be trapped in risky positions they could not unwind.

To address the impact of this issue on derivatives' pricing, we consider a discretetime setting such that transactions are not possible within a subset of points in time. Although clearly very stylized, the advantage of this setting is that it incorporates in a very simple way the notion of market illiquidity as the absence of immediacy. Under such illiquidity we say that markets are dry. In this framework, dryness changes what is otherwise a complete market into a dynamically incomplete market. This was also the approach in A maro de Matos and Antao (2001) when characterizing the speci ${ }^{-}$c superreplication bounds for options in such markets and its implications. We further extend this setting by assuming that transactions occur at each point in time with a given probability, re $^{\circ}$ ecting a more realistic ex-ante uncertainty about the market.

As stressed above, there is not a unique arbitrage-free value for a derivative under market incompleteness. However, for any given derivative, portfoli os can be found that have the same payo ${ }^{\circ}$ as the derivative in some states of nature, and higher payo ${ }^{\circ}$ in the other states. Such portfolios are said to be superreplicating. Holding such a portfolio should be worth more than the option itself and therefore, the value of the cheapest of such portfolios should be seen as an upper bound on the selling value of the option. Similarly, a lower bound for the buying price can be constructed. The nature of the superreplicating bounds is well characterized in the context of incomplete markets in the papers by EI Karoui and Quenez $(1991,1995)$, Edirisinghe, Naik and Uppal (1993) and K aratzas and K ou (1996). The superreplicating bounds establish the limits of the interval for arbitrage-free value of the 
option. If the price is outside this range, then a positive pro $^{-} t$ is attainable with probability one. Therefore, the equilibrium prices at which the derivative is transacted should lie between those bounds.

M ost of the times, however, these superreplicating bounds are trivial, in the sense that they are too broad, not allowing a useful characterization of equilibrium prices' vicinity. As an alternative, Bernardo and Ledoit (2000) propose a utility-based approach, restricting the no-arbitrage condition to rule out investment opportunities o Bering high gain-loss ratios, where gain (loss) is the expected positive (negative) part of excess payo ${ }^{\circ}$. In this way, narrower bounds are obtained. A nalogously, Cochrane and Sa\$-R equejo (2000) also restrict the no-arbitrage condition by not allowing tran sactions of I good deals", i.e. assets with very high Sharpe ratio. Following Hansen and J agannathan (1991), they show that this restriction imposes an upper bound on the pricing kernel volatility and leads to narrower pricing implications when markets are incomplete.

Given a set of pricing kernels compatible with the absence of arbitrage opportunities, Cochrane and S\&a-R equejo exclude pricing kernels implying very high Sharpe ratios, whereas Bernardo and Ledoit exclude pricing kernels implying very high gain-loss ratios for a benchmark utility. Notice that, for a di Rerent utility, B ernardo and Ledoit would exclude a di Rerent subset of pricing kernels, for the same levels of acceptable gain-l oss ratios. Also notice that the interval designed by Cochrane and S\&a-Requejo is not necessarily arbitrage free, and therefore does not necessarily contain the equilibrium price.

In order to avoid ad-hoc thresholds in either Sharpe or gain-loss ratios, or to make some parametric assumptions about a benchmark pricing kernel, as in B ernardo and Ledoit (2000), the work of Bondarenko (2003) introduces the notion of statistical arbitrage opportunity, by imposing a weak assumption on a functional form of admissible pricing kernels, yielding narrower pricing implications as compared to the superreplication bounds. A statistical arbitrage opportunity is characterized as a zero-cost trading strategy for which (i) the expected payo ${ }^{\circledR}$ is positive, and (ii) the conditional expected payo ${ }^{\circledR}$ in each " nal state of the economy is non negative. Unlike a pure arbitrage opportunity, a statistical arbitrage opportunity may allow for negative payo ${ }^{\circledR S}$, provided that the average payo ${ }^{\circledR}$ in each ${ }^{-}$nal state is nonnegative. In particular, ruling out statistical arbitrage opportunities imposes a novel martingale-type restriction on the dynamics of securities prices. The important properties of the restriction are that it is model-free, in the sense that it requires no parametric assumptions about the true equilibrium model, and continues to hold when investors' beliefs are mistaken. A lthough B on- 
darenko's interval can be shown to be in the arbitrage-free region, it does not necessarily contain the equilibrium value of the derivative.

In this paper we extend the notion of statistical arbitrage opportunity to the case where the underlying asset can be transacted at each point in time with a given probability, and compare the statistical arbitrage-free bounds with the superreplication bounds. We show that the statistical arbitrage-free interval is narrower than the pure arbitrage bounds, and show also that, for su \pm ciently incomplete markets (probability not too close to 1 ), thestatistical arbitrage interval contains the reservation price of the derivative. We also provide examples that allow comparison with the results of Cochrane and Sa\$-R equejo (2000) and discuss the comparison with B ernardo and Ledoit (2000).

This paper is organized as follows. In section 2, the model is presented and the pure arbitrage results are derived. In section 3 the notion of statistical arbitrage in the spirit of Bondarenko (2003) is de- ned. In section 4 , the main results are presented. In Section 5 we ${ }^{-}$rst characterize the reservation prices and then show that, in a su \pm ciently dry market, they are contained in the statistical arbitrage interval. In Section 6 we illustrate how the statistical arbitrage-free interval somehow dominates alternative intervals provided in the literature. In section 7 some numerical examples are presented in order to illustrate some important properties of the bounds. In the last section several conclusions are presented.

\section{The Model}

Consider a discrete-time economy with $\mathrm{T}$ periods, with a risky asset and a riskless asset. At each point in time the price of the risky asset can be multiplied either by $U$ or by $D$ to get the price of the next point in time. Equilibrium requires that $U>R>D$, where $R$ denotes oneplus the risk-free interest rate. At time $\mathrm{t}=0$ and $\mathrm{t}=\mathrm{T}$ transactions are certainly possible. However, at $t=1 ;: ;: ; \mathrm{T}$ i 1 there is uncertainty about the possibility of transaction of the risky asset. Transactions will occur with probability $p$ at each of these points in time. A European Derivative with maturity $T$ is considered.

Consider the Binomial tree process followed by the price of the risky asset. Let the set of nodes at date $t$ be denoted by $\mathrm{I}_{\mathrm{t}}$; and let each of the $t+1$ elements of $I_{t}$ be denoted by $i_{t}=1 ;:: ; ; t+1$. For any $t^{0}<t$, let $I_{t^{0}}^{i_{t}}$ denote the set of all the nodes at time $t^{0}$ that are predecessors of a given node $i_{t}$ : A path on the event tree is a set of nodes $w=\left[t 2 f 0 ; 1 ; \ldots ; ; T_{g} i_{t}\right.$ such 
that each element in the union satis es $i_{t_{i}} 2 I_{t_{i} 1}^{i_{t}}$ : Let - denote the set of all paths on the event tre:

The payo ${ }^{\circledR} S$ of a E uropean derivative, at each terminal node, will be denoted $G_{T}^{i_{T}}$ : At each node $i_{t}$; the stock price is given by $S_{t}^{i_{t}}=U^{t+1_{i}} i_{t} D^{i_{t} i}{ }^{1} S_{0}$ : Moreover, at each node $i_{t}$; there is a number $\phi_{t}{ }_{t}$ representing the number of shares bought (or sold, if negative), and a number $B_{t}^{i t}$ denoting the amount invested (or borrowed, if negative) in the risk-free asset. Hence, at there are $t+1$ values of $\phi_{t}^{i t}$, composing a vector $\phi_{t}^{\prime}\left(\phi \frac{1}{t} ;::: ; \phi_{t}^{t+1}\right) 2 R^{t+1}$ : Similarly, we construct the vector $\mathrm{B}_{\mathrm{t}}^{\prime}\left(\mathrm{B}_{\mathrm{t}}^{1} ;::: ; \mathrm{B}_{\mathrm{t}}^{\mathrm{t}+1}\right) 2 \mathrm{R}^{\mathrm{t}+1}$ :

De $e^{-}$nition $1 \mathrm{~A}$ trading strategy is a portfolio process $\mu_{t}=\left(\$ t ; B_{t}\right)$; composed of $\phi \mathrm{t}$ units of the risky asset and an amount $B_{t}$ invested in the riskless asset, such that the portfolio's cost is $\phi t S_{t}+B_{t}$ for $t=0 ; 1 ;::: T$ i 1 :

In order to ${ }^{-}$nd the upper (lower) bound of the arbitrage free range of variation for the value of a European derivative we consider a "nancial institution that wishes to be fully hedged when selling (buying) that derivative. The objective of the institution is to minimize (maximize) the cost of replicating the exercise value of the derivative at maturity. The value determined under such optimization procedure avoids what is known as arbitrage opportunities, re $\mathrm{e}^{\circ}$ ecting the possibility of certain pro ${ }^{-}$ts at zero cost.

This section is organized as follows. We ${ }^{-}$rst characterize the upper bound, and then the lower bound for the interval of no-arbitrage admissible prices. For each bound, we ${ }^{-}$rst deal with the complete market case, and then with the fully incomplete market case, " nally introducing random incompleteness.

\subsection{The upper bound in the case $p=0$ and $p=1$ :}

First, we present the well-known case where $p=1$. The usual de ${ }^{-}$nition of an arbitrage opportunity in our economy is as follows.

De $e^{-}$nition 2 (P ure Arbitrage in the case $p=1$ ) In this economy, an arbitrage opportunity is a zero cost trading strategy $\mu_{\mathrm{t}}$ such that

1. the value of the portfolio is positive at any ${ }^{-}$nal node, i.e., $\phi_{T_{i}}^{i_{T}}{ }_{1}^{1} S_{T}^{i_{T}}+$ $R B_{T_{i}{ }_{1}}^{i_{T}}, 0$; for any $i_{T_{i}} 12 I_{T_{i} 1}^{i_{T}}$ and all $i_{T} 2 I_{T}$; and

2. the portfolio is self- ${ }^{-}$nancing, i.e., $\Phi_{t_{i}{ }_{1}}^{i_{t_{i}}} S_{t}^{i_{t}}+R B_{t_{i}}^{i_{t_{i}} 1}, \Phi_{t}^{i_{t}} S_{t}^{i_{t}}+B_{t}^{i_{t}}$; for any $\mathrm{i}_{\mathrm{t}_{\mathrm{i}}} 2 \mathrm{I}_{\mathrm{t}_{\mathrm{i}}}^{\mathrm{i}_{\mathrm{t}}} ;$ all $\mathrm{i}_{\mathrm{t}} 2 \mathrm{I}_{\mathrm{t}}$ and all $\mathrm{t} 2 \mathrm{f0} ; \ldots ;: \mathrm{T} \mathrm{i} 1 \mathrm{~g}$ : 
The upper bound for the value of the European option is the maximum value for which the derivative can be transacted, without allowing for arbitrage opportunities. This is the value of the cheapest portfolio that the seller of the derivative can buy in order to completely hedge his position against the exercise at maturity, without the need of additional " nancing at any rebalancing dates. Hence, for $p=1$; the upper bound is $\mathrm{C}_{\mathrm{u}}^{1}$; given by

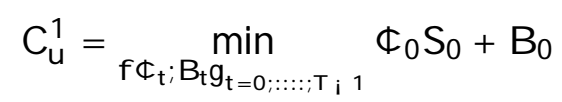

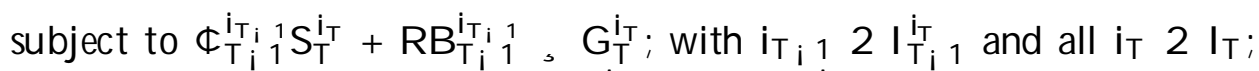
and the self- ${ }^{-}$nancing constraints $\phi_{t_{i}}^{i_{t_{i}}} S_{t}^{i_{t}}+R B_{t_{i} 1}^{i_{t_{i}} 1}, \phi_{t}^{i_{t}} S_{t}^{i_{t}}+B_{t}^{i_{t}}$; for all

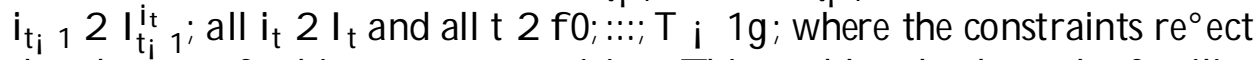
the absence of arbitrage opportunities. This problem leads to the familiar result

$$
C_{u}^{1}={\frac{1}{R^{T}}}_{j=0}^{X \mu_{T}{ }^{\eta} \mu_{R_{i} D}{ }^{\eta_{j}} \mu_{U_{i} D}} \frac{U_{i} R}{U_{i} D}{ }^{\eta_{T i} j} G_{T}^{T+l_{i} j}:
$$

Consider now the case where $p=0$. In this case, the notion of a trading strate ${ }_{T}$ gy satisfying the self- ${ }^{-}$nancing constraint is innocuous, since the portfolio $\mu_{t}$ cannot be rebalanced during the life of the option. Under the absence of arbitrage opportunities, the upper bound for the value is $\mathrm{C}_{\mathrm{u}}^{0}$ satisfying

$$
\mathrm{C}_{\mathrm{u}}^{0}=\min _{\mathrm{f} \phi_{0} ; \mathrm{B}_{0} \mathrm{~g}} \phi_{0} \mathrm{~S}_{0}+\mathrm{B}_{0}
$$

subject to $\phi_{0} S_{T}^{i_{T}}+R^{\top} \phi 0, G_{T}^{i_{T}}$; for all $i_{T} 2 I_{T}$ : In this case, the bound $\mathrm{C}_{\mathrm{u}}^{0}$ can be shown to splve the maximization problem on a set of positive constants $f \#_{T} g$; with ${ }_{i_{T}=1}^{+1} \#_{T}=1$;

$$
\mathrm{C}_{\mathrm{u}}^{0}=\max _{\#_{\mathrm{T}}} \frac{1}{\mathrm{R}^{\top}} \stackrel{\mathrm{i}_{\mathrm{T}}=1}{\mathrm{~T}+1} \#_{\mathrm{T}} \mathrm{G}_{\mathrm{T}}^{\mathrm{i}_{\mathrm{T}}}
$$

subject to

$$
\mathrm{S}_{0}={\frac{1}{\mathrm{R}^{\top}}}^{\mathrm{X}} \underset{\mathrm{i}+1}{\mathrm{i}=1} \#_{\mathrm{T}} \mathrm{H}_{\mathrm{T}}^{\mathrm{i}_{\mathrm{T}}}
$$

For instance, if a call option with exercise $\mathrm{K}$ is considered, we have ${ }^{1}$

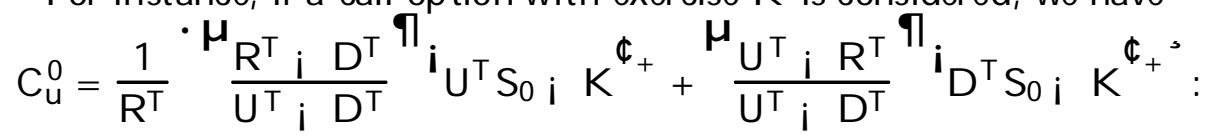

\footnotetext{
${ }^{1}$ See A maro de Matos and Antao (2001).
} 


\subsection{The lower bound in the case $p=0$ and $p=1$ :}

The lower bound for the value of an A merican derivative is the minimum value for which the derivative can be transacted without allowing for arbitrage opportunities. This is the value of the most expensive portfolio that the buyer of the option can sell in order to be fully hedged, and without the need of additional " nancing at rebalancing dates.

For $p=1$, the lower bound for the value of the derivative under the absence of arbitrage opportunities is thus $\mathrm{C}_{1}^{1}$; given by

$$
\mathrm{C}_{\mathrm{l}}^{1}=\max _{\mathrm{f} \notin \mathrm{t} ; \mathrm{B}_{\mathrm{t}} \mathrm{g}_{\mathrm{t}=0 ; \cdots ; ; \mathrm{T} ; 1}} \phi \mathrm{O}_{0}+\mathrm{B}_{0}
$$

subject to $\phi \mathrm{T}_{T_{i}}^{i_{1}}{ }_{1} S_{T}^{i_{T}}+R B_{T_{i}}^{i_{T}}{ }_{1} \cdot G_{T}^{i_{T}}$, with $i_{T_{i}} 12 I_{T_{i} 1}^{i_{T}}$ and all $i_{T} 2 I_{T}$; and the self- ${ }^{-}$nancing constraints $\phi_{t_{i} 1}^{i_{t_{i}}{ }_{1}} S_{t}^{i_{t}}+R B_{t_{i} 1}^{i_{t_{i} 1}} \cdot \phi_{t}^{i_{t}} S_{t}^{i_{t}}+B_{t}^{i_{t}}$; for all $\mathrm{i}_{\mathrm{t}_{\mathrm{i}} 1} 2 \mathrm{I}_{\mathrm{t}_{\mathrm{i}} 1}^{\mathrm{it}_{\mathrm{t}}}$; all $\mathrm{i}_{\mathrm{t}} 2 \mathrm{I}_{\mathrm{t}}$ and all $\mathrm{t} 2 \mathrm{f} 0 ; \ldots ; \mathrm{T} \mathrm{i} 1 \mathrm{~g}$; where the constraints re ect the absence of arbitrage opportunities $T$ his problem leads to the familiar result

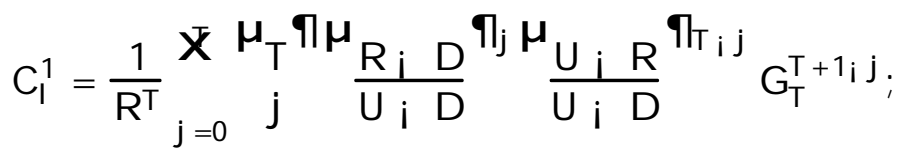

that coincides with the solution obtained for $\mathrm{C}_{\mathrm{u}}^{1}$ :

In the case where $p=0$; the lower bound for the value of the derivative is $C_{\mid}^{0}$; satisfying

$$
C_{l}^{0}=\max _{f \notin 0 ; B_{0} g} \notin S_{0}+B_{0}
$$

subject to $₫{ }_{0} S_{T}+R^{\top} B_{\beta} \cdot G_{T}^{i_{T}}$ : A s above, it follows that, for a set of positive constants $f \#_{T} g$; with ${ }_{i_{T}=1}^{T+1} \#_{T}=1$; this bound is given by

$$
C_{u}^{0}=\min _{\#_{T}}{\frac{1}{R^{\top}}}^{X} i_{i_{T}=1}^{T+1} \#_{T} G_{T}^{i_{T}}
$$

subject to

$$
S_{0}={\frac{1}{R^{\top}}}^{X} \underbrace{T+1}_{i_{T}=1} \#_{T} S_{T}^{i_{T}}:
$$


In the case of a call option with exercise $K$, we have ${ }^{2}$

$$
\begin{aligned}
& C_{l}^{0}=\frac{1}{R^{\top}} \frac{R^{\top} i U^{\top i}{ }^{(i+1)} D^{i+1}}{U^{\top}{ }^{i} D^{i} i U^{\top i}(i+1) D^{i+1}}{ }^{i} U^{\top}{ }^{i} D^{i} S_{0} i K^{\phi_{+}}
\end{aligned}
$$

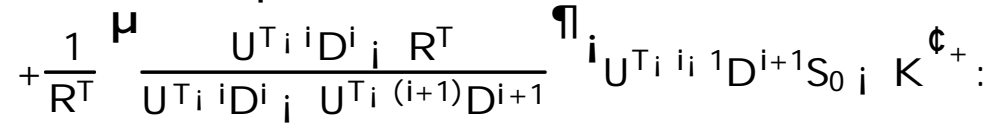

where $i$ is de $e^{-}$ned as the unique integer satisfying $U^{n_{i}}{ }^{(i+1)} D^{i+1}<R^{n}<$

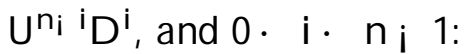

\subsection{The Bounds on Probabilistic M arkets}

In the aforementioned cases we considered the cases where either $p=0$ or $p=1$. However, if $p$ is not equal to neither 0 nor 1 , the formulation has to be adjusted. If the risky asset can be transacted with a given probability p2 $(0 ; 1)$, then the usual de nition of arbitrage opportu nity reads as follows.

De- nition 3 (P ure Arbitrage for p2 $(0 ; 1)$ ) In this economy, an arbitrage opportunity is a zero cost trading strategy such that

1. the value of the portfolio is positive at any " nal node, i.e.,

$$
\phi{ }_{t}^{i_{t}} S_{T}^{i_{T}}+R^{T i}{ }^{t_{t}} B_{t}^{i_{t}}, 0 ;
$$

$\mathrm{i}_{\mathrm{t}} 2 \mathrm{I}_{\mathrm{t}}^{\mathrm{i}_{\mathrm{T}}}$ and all $\mathrm{i}_{\mathrm{T}} 2 \mathrm{I}_{\mathrm{T}}$; and the self- ${ }^{-}$nancing constraints

2. the portfolio is self- ${ }^{-}$nancing, i.e.,

$$
\phi_{t_{i} j}^{i_{t_{j} j}} S_{t}^{i_{t}}+R^{j} B_{t_{i} j}^{i_{t_{i} j}}, \phi_{t}^{i_{t}} S_{t}^{i_{t}}+B_{t}^{i_{t}}
$$

for all $i_{t_{i} j} 2 I_{t_{i} j}^{i_{t}} ;$ all $i_{t} 2 I_{t}$ and all t $2 \mathrm{f} 0 ;:: ; ; T$ i $1 \mathrm{~g}$ :

The upper bound $C_{u}^{p}$ is the solution of the following problem:

$$
C_{\mathrm{u}}^{\mathrm{p}}=\min _{\mathrm{f} \notin \mathrm{t} ; \mathrm{B} t \mathrm{~g}_{\mathrm{t}=0 ; \cdots ; \mathrm{T}_{\mathrm{i}} 1}} \$ 0 \mathrm{~S}_{0}+\mathrm{B}_{0}
$$

where $\phi_{t} ; B_{t} 2 R^{t+1} ; t=0 ;:: ; T ; 1$; subject to the superreplicating condi-

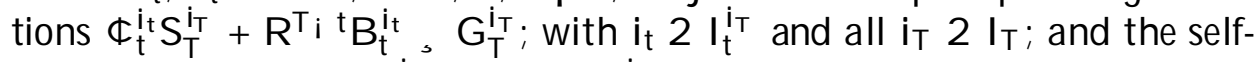
- nancing constraints $\phi_{t_{i} j}^{i_{t_{j} j}} S_{t}^{i_{t}}+R^{j} B_{t_{i} j}^{i_{t_{i} j}}, \phi_{t}^{i_{t}} S_{t}^{i_{t}}+B_{t}^{i_{t}}$ for all $i_{t_{i} j} 2 I_{t_{i} j}^{i_{t}}$; all $i_{t} 2 I_{t}$ and all t 2 f0;:::; $T_{i} 1 g$ :

On the other hand, the lower bound $C_{\mid}^{p}$ solves the following problem:

\footnotetext{
${ }^{2}$ See A maro de Matos and Antao (2001).
} 


$$
C_{l}^{p}=\max _{f \notin t ; B t g_{t=0 ; \cdots ; T_{i} 1}} \notin 0 S_{0}+B_{0}
$$

where $\phi_{t} ; B_{t} 2 R^{t+1} ; t=0 ;:: ; T$ i 1 ; subject to the conditions $\phi_{t}^{i_{t}} S_{T}^{i_{T}}+$

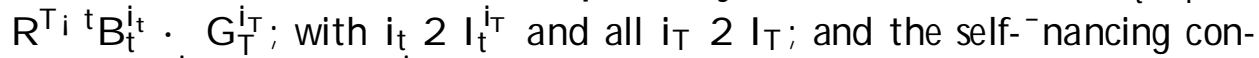

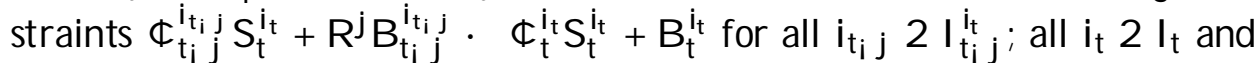
all t 2 f0;::.; T i 19 :

Notice that the constraints in the above optimization problems are implied by the absence of arbitrage opportunities and do not depend on the probability $p:{ }^{3}$ Therefore, neither $C_{u}^{p}$ nor $C_{l}^{p}$ will depend on $p:$ We are now in conditions to relate these values to $\mathrm{C}_{\mathrm{u}}^{0}$ and $\mathrm{C}_{\mathrm{l}}^{0}$ as follows.

Theorem 4 For p $2(0 ; 1)$ the upper and lower bound for the prices above do not depend on $\mathrm{p}$ : The optimization problems above lead to the same solutions as when $p=0$ :

P roof. Consider ${ }^{-} r s t$ the case of the upper bound. The constraints characterizing $\mathrm{C}_{\mathrm{u}}^{\mathrm{p}}$ include all the constraints characterizing $\mathrm{C}_{\mathrm{u}}^{0}$ : T hus, $\mathrm{C}_{\mathrm{u}}^{\mathrm{p}}$, $\mathrm{C}_{\mathrm{u}}^{0}$. Now, let $\phi_{0}^{0}$ and $\mathrm{B}_{0}^{0}$ denote the optimal values invested, at time $t=0$; when $p=0$. The trading strategy $\phi_{t}^{p}=\phi_{0}^{0}$ and $B_{t}^{p}=R^{t} B_{0}^{0}$, for all $t=1 ;:: ; T_{i} 1$, is an admissible strategy for any given $p$, hence $C_{u}^{p}=C_{u}^{0}$ : The case of the lower bound is analogous.

The intuition for this result is straightforward. The upper (lower) bound of the European derivative remains the same as when $p=0$; because with probability 1 i $\mathrm{p}$ it would not be possible to transact the stock at each point in time. In order to be fully hedged, as required by the absence of arbitrage opportunities, the worse scenario will be restrictive in spite of its possibly low probability. The fact that no intermediate transactions may occur dominates all other possibilities.

The above result is strongly driven by the de ${ }^{-}$nition of arbitrage opportunities. Nevertheless, if this notion is relaxed in an economic sensible way, a narrower arbitrage-free range of variation for the value of the European derivative may be obtained, possibly depending now on $\mathrm{p}$ : T his is the subject of the rest of the paper.

\footnotetext{
${ }^{3} \mathrm{~T}$ his happens since, in order to have an arbitrage opportunity, we must ensure that, whether market exists or not at each time $2 \mathrm{fl} ;:: ; ; \mathrm{T} ; \mathrm{lg}$; the agent will never lose wealth. Therefore, the optimization problem cannot depend on $\mathrm{p}$ :
} 


\section{Statistical A rbitrage Opportunity}

Consider theeconomy described in the previous section. Let $T_{p}=f 1 ; \ldots ; ; i \quad 1 g$ denote the set of points in time. At each of these points there is market with probability $p$; and there is no market with probability $1 \mathrm{i} p$ : The existence (or not) of the market at time $t$ corresponds to the realization of a random variable $y_{t}$ that assumes the value 0 (when there is no market) and 1 (when there is market). This random variable is de ned for all t $2 T_{p}$ and it is assumed to be independent of the ordinary source of uncertainty that generates the price process. We can therefore talk about a market existence process. In order to construct one such process, let us start with the state space. Let \# $\left(T_{p}\right)$ denote the number of points in $T_{p}$ : At each of these points, market may either exist or not exist, leading to $2^{\#\left(T_{p}\right)}$ possible states of nature. We then have the collection of possible states of nature denoted by $\hat{\imath}=f v_{i} g_{i=1 ; \ldots ; 2^{\#}}\left(T_{p}\right)$; each $v_{i}$ corresponding to a distinct state. Moreover, let $\hat{F^{\prime}}=\hat{F_{1}} ;::: ; \hat{F_{T}}$; where $\hat{F_{t}}$ is the $3 / 4$ algebra generated by the random variable $y_{t}$. Let $p_{y}$ be the probability associated with the random variable $y_{t}: F$ or all t $2 T_{p}$; we have $p_{y}\left(y_{t}=1\right)=p$ and $p_{y}\left(y_{t}=0\right)=1 ; p$ :

\subsection{The expected value of a portfolio}

We now construct a random variable that allows to construct the expected future value of a portfolio in this setting. For $\mathrm{t}<\mathrm{t}^{0}$, let $\mathrm{x}_{\mathrm{t} ; \mathrm{t}}$ be a random variable identifying the last time that transactions take place before date $t^{0}$, given that we are at time $t$; and transactions are curregtly possible. Let $\hat{-t}$ be the subset of $\hat{\wedge}$ such that $\hat{{ }_{\mathrm{t}}}=\mathrm{v}_{\mathrm{i}} \hat{2} \hat{-}: \mathrm{y}_{\mathrm{t}}\left(\mathrm{v}_{\mathrm{i}}\right)=1$ : Then,

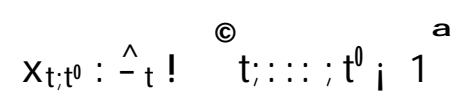

Let $p_{\mathrm{x}_{\mathrm{t} ; \mathrm{t}}}$ be the probability associated with $\mathrm{x}_{\mathrm{t} ; \mathrm{t}}$ : Then,

$$
p_{x_{t ;} t^{0}}{ }^{i} x_{t ; t^{0}}=t^{\phi}=(1 ; p)^{t^{0} t_{i} 1} \text { : }
$$

Moreover, for a given s $2(t ; t)$;

$$
p_{x t ; 0^{0}}{ }^{i} x_{t ; t^{0}}=s^{\phi}=p(1 ; p)^{t^{0_{i}} s_{i} 1} \text { : }
$$

Also note that

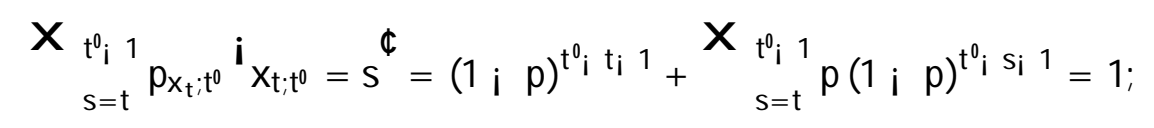


as it should.

Consider a given trading strategy $\left(\phi t ; B_{t}\right)_{t=0 ; \ldots ; T ;}$; where $\left(\phi t ; B_{t}\right)$ $\left(\phi+i_{t}^{i} ; B_{t}^{i_{t}}\right)_{i_{t} 2 I_{t}}$ is a $(t+1)$ i dimensional vector. Consider a given path $w$

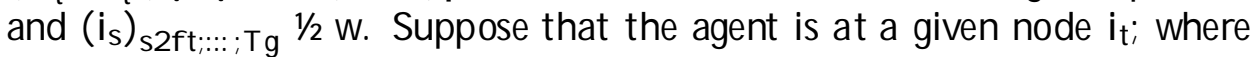
rebalancing is possible. As there is uncertainty about the existence of market at the future points in time, there is also uncertainty about the portfolio that the agent will be holding at a future node $i_{t}$ : In fact, the poptfolio at $\mathrm{i}_{\mathrm{t}}$ may be any of $\phi_{\mathrm{t}}^{\mathrm{i}_{\mathrm{t}}} ; \mathrm{B}_{\mathrm{t}}^{\mathrm{i}_{\mathrm{t}}} ; \phi_{\mathrm{t}+1}^{\mathrm{i}_{\mathrm{t}+1}} ; \mathrm{B}_{\mathrm{t}+1}^{\mathrm{i}_{\mathrm{t}+1}} ;::: ;$ or $\phi_{\mathrm{t}_{\mathrm{i}}{ }_{1}}^{\mathrm{i}_{\mathrm{i} \mathrm{q}_{1}}} ; \mathrm{B}_{\mathrm{t} 0_{\mathrm{i}}{ }_{1}}^{\mathrm{i}_{\mathrm{t}} \mathrm{O}_{1}}$; where

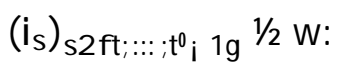

Clearly, the expected value of a given trading strategy at node $\mathrm{i}_{\mathrm{t}}$, given that we are at node $\mathrm{i}_{\mathrm{t}}$; is

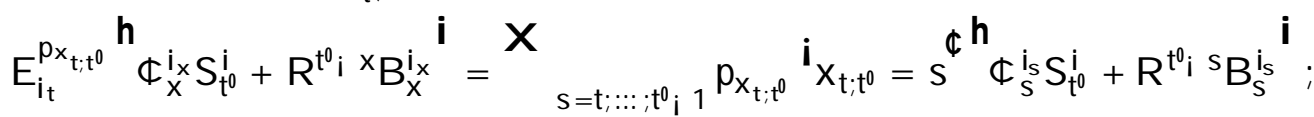

where we use $\mathrm{x}$ to short notation for $\mathrm{x}_{\mathrm{t} ; \mathrm{t}} \mathrm{t}$ :

\subsection{Statistical versus pure arbitrage}

A pure arbitrage opportunity is a zero-cost portfolio at time $t$, such that the value of each possible portfolio at node $i_{T}$ is positive, i.e.,

$$
\phi_{t+j}^{i_{t+j}} S_{T}^{i_{T}}+R^{T_{i} t_{i} j} B_{t+j}^{i_{t+j}}, 0
$$

for all $i_{t+j}$ such that $i_{t}$ is a predecessor, $j=0 ; 1 ;::: ; \mathrm{T}_{\mathrm{i}} \mathrm{t}_{\mathrm{i}} 1$ and

$$
E_{i_{t}}^{p_{x_{t} ; T}}{ }^{h}{ }_{x}^{i_{x}} S_{T}^{i_{T}}+R^{T_{i} \times} B_{x}^{i_{x}}>0 \text {; }
$$

together with the self-- nancing constraints

$$
\phi_{t}^{i_{t}} S_{t+j}^{i_{t+j}}+R^{j i}{ }^{t_{t}} B_{t}^{i_{t}}, \phi_{t+j}^{i_{t+j}} S_{t+j}^{i_{t+j}}+B_{t+j}^{i_{t+j}}
$$

for all $i_{t+j}$ such that $i_{t}$ is a predecessor, and $j=1 ;::: ; T_{i} t_{i} 1$ :

If statistical arbitrage is considered, however, an arbitrage opportunity requires only that, at node $i_{T}$; the expected value of the portfolio at $T$ is positive,

$$
E_{i t}^{p_{x_{t} ; T}} \stackrel{h}{\phi}{ }_{x}^{i_{x}} S_{T}^{i_{T}}+R^{T_{i} \times} B_{x}^{i_{x}}{ }^{i}, 0 ;
$$

together with weaker self- ${ }^{-}$nancing conditions. Let us regard these latter conditions in some detail. 
Suppose that we are at a given node $\mathrm{i}_{\mathrm{t}}$ : If these is market at the next point in time we then have, for sure, the portfolio $\phi_{t}^{i_{t}} ; B_{t}^{i_{t}}$ at time $t+1$. Hence, if node $i_{t+1}$ is reached, the self-' nancing condition is

$$
\phi_{t}^{i_{t}} S_{t+1}^{i_{t+1}}+R B_{t}^{i_{t}} i^{3} \quad \phi_{t+1}^{i_{t+1}} S_{t+1}^{i_{t+1}}+B_{t+1}^{i_{t+1}}, 0
$$

Consider now that $t+2$ is reached. At time $t$ there is uncertainty about the existence of the market at time $t+1$ : Hence $_{\beta}$ at time $t+2$ we can either have the portfolio $\phi_{t}^{i_{t}} ; B_{t}^{i_{t}}$ or the portfolio $\phi_{t+1}^{i_{t+1}} ; B_{t+1}^{i_{t+1}}$ : Under the concept of statistical arbitrage, we want to ensure that, in expected value, we are not going to lose at node $\mathrm{i}_{\mathrm{t}+2}$ : Hence, the self- nancing condition becomes

$$
X_{s=t ; t+1} p_{x t ; t+2}\left(x_{t ; t+2}=s\right)^{3} \phi_{s}^{i_{s}} S_{t+2}^{i_{t+2}}+R^{t+2 i} s_{s}^{i_{s}}{ }^{3}, \phi_{t+2}^{i_{t+2}} s_{t+2}^{t_{+2}}+B_{t+2}^{i_{t+2}}
$$

More generally, for any $t$ at which transaction occurs and $t<t^{0}<\mathrm{T}$; the statistical self- ${ }^{-}$nancing condition becomes

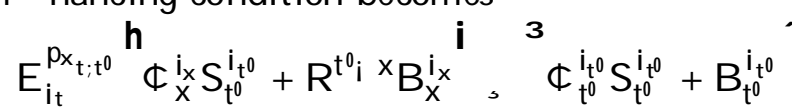

De nition $5^{4}$ A statistical arbitrage opportunity is a zero-cost trading strategy for which

1. At any node $i_{t}$, the expected value of the portfolio at any ${ }^{-}$nal node is positive, i.e.,

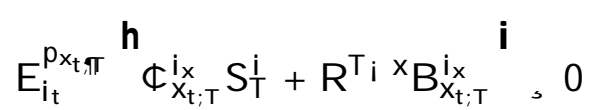

${ }^{4} \mathrm{~T}$ his notion of Arbitrage Opportunity is in the spirit of Bondarenko (2003). In his de ${ }^{-}$nition 2, a Statistical A rbitrage Opportunity (SAO) is de ${ }^{-}$ned as a zero-cost trading strategy with a payo ${ }^{\circledR} Z_{T}=Z\left(F_{T}\right)$, such that

(i) $E\left[Z_{T} j F_{0}\right]>0$; and

(ii) $\mathrm{E}\left[\mathrm{Z}_{\mathrm{T}} \mathrm{jF} \mathrm{F}_{0}\right.$ » $\left.\mathrm{T}_{\mathrm{T}}\right], 0$; for all $»_{\mathrm{T}}$;

where $»_{t}$ denotes the state of the $\mathrm{N}$ ature at time $t$; and $\mathrm{F}_{t}=\left(»_{1} ;::: ; »_{t}\right)$ is the market information set, with $\mathrm{F}_{0}=\mathrm{A}$. A lso, the second expectation is taken at time $\mathrm{t}=0$ and is conditional to the terminal state " $T$. However, notice that eliminating SAO's at time $\mathrm{t}=0$ does not imply the absence of SAO's at future times $\mathrm{t} 2[1 ; \mathrm{T} ; 1]$. Hence, in order to incorp orate a dynamically consistent absence of SAO's, we re ne the de nition of a SAO as a zero-cost trading strategy with a payo ${ }^{\circledR} Z_{T}=Z\left(F_{T}\right)$, such that

(i) $\mathrm{E}\left[\mathrm{Z}_{\mathrm{T}} \mathrm{j} \mathrm{F}_{0}\right]>0$; and

(ii) $E\left[Z_{T} j F_{t} ; »_{T}\right], 0$; for all $»_{T}$ and all t $2[0 ; T ; 1]$ : 
for any $\mathrm{i}_{\mathrm{t}} 2 \mathrm{I}_{\mathrm{t}}$ and $\mathrm{t} 2 \mathrm{f} 0 ; 1 ;::: ; \mathrm{T} \mathrm{i} \mathrm{g}$; and

2. The portfolio is statistical ly self-" nancing, i.e:,

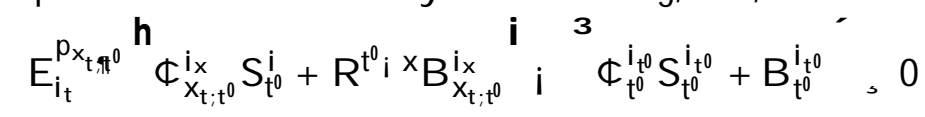

for any $i_{t} 2 I_{t} ; t^{0}>t, t 2 f 0 ; 1 ;::: ; T ; 2 g$ and $t^{0} 2 f 1 ;::: ; T ; 1 g:$

The two de nitions of arbitrage are related in the following.

Theorem 6 If there are no statistical arbitrage opportunities, then there are no pure arbitrage opportunities.

P roof. If there is a pure arbitrage opportunity then the inequalities present in the de ${ }^{-}$nition of arbitrage opportunity, de ${ }^{-}$nition 3 , are respected. Hence, as these expressions are the terms under expectation in the de ${ }^{-}$nition of Statistical A rbitrage opportunity, presented in de nition 5, there is also a statistical arbitrage opportunity.

The set of portfolios that represent a pure arbitrage opportunity is a subset of the portfolios that represent a statistical arbitrage opportunity, i.e., there are portfolios that, in spite of not being a pure arbitrage opportunity, represent a statistical arbitrage opportunity.

In order to have a statistical arbitrage opportunity it is not necessary (although it is su \pm cient) that the value of the portfolio at the ${ }^{-}$nal date is positive. It is only necessary that, for all $t$, the expected value of the portfolio at the " nal date is positive.

Consider now the self- ${ }^{-}$nancing conditions under statistical arbitrage. When rebalancing the portfolio it is not necessary (although it is su \pm cient) that the value of the new portfolio is smaller than the value of the old one. This happens because future rebalancing is uncertain, leading to uncertainty about the portfolio that the agent will be holding in any future moment. In order to avoid a statistical arbitrage opportunity it is only necessary that the expected value of the portfolio at a given point in time is larger than the value of the rebalancing portfolio.

Finally, notice that the concept of statistical arbitrage opportunity reduces to the usual concept of arbitrage opportunity in the limiting case $p=0$ :

\subsection{Augmented measures}

For technical reasons, we now de ne an augmented probability space $\mathrm{Q}$ on - . In order to do that, we de ne a semi path $m$ from $i_{t}$ to $i_{t}$, which is a set 
of nodes $m=\left[k_{k 2 f t ;: ;: ; t g} i_{k}\right.$ such that $i_{k} 2 I_{k}^{i_{k+1}}$ : Let $-{ }_{i_{t}}^{+} ; i_{t}$ denote the set of semipaths from $i_{t}$ to $i_{t} 0$ :

$\mathrm{Re}^{-}$nition $7 \mathrm{An}$ augmented probability space in - is a set of probabilities $\mathrm{q}_{\left(\mathrm{i}_{\mathrm{t}} ; \mathrm{t}\right)}^{\left(\mathrm{i}_{0} ; \mathrm{T}\right) ; \mathrm{m}}$ such that $\mathrm{i}_{\mathrm{t}} 2 \mathrm{I}_{\mathrm{t}} ; \mathrm{m} 2-{ }_{\mathrm{i}_{\mathrm{t}} ; \mathrm{i}_{\mathrm{T}}}^{+}, \mathrm{t}=0 ;::: ; \mathrm{T}$ and

$$
\begin{array}{llllll}
X & X^{1} & 1 X & X & q_{\left(i_{t} ; t\right)}^{\left(i_{T} ; T\right)}=1 ;
\end{array}
$$

$\mathrm{De}^{-}$nition $8 \mathrm{~A} \mathrm{modi}^{-}$ed martingale probability measure is an augmented probability measure Q 2 Q which satis ${ }^{-}$es

(i)

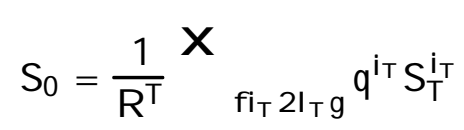

where

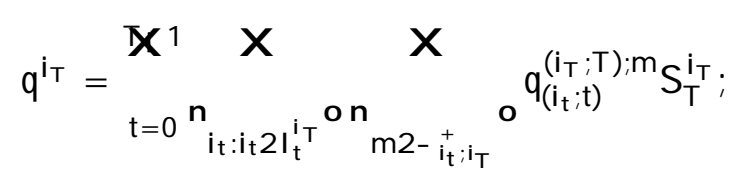

(ii)

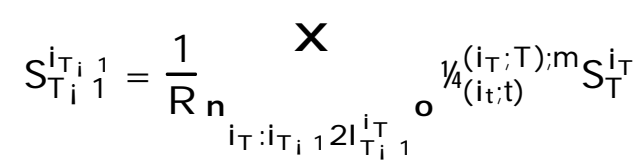

with

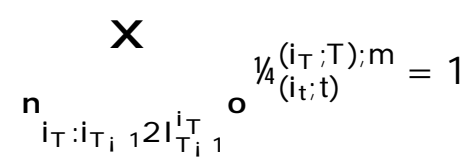

and

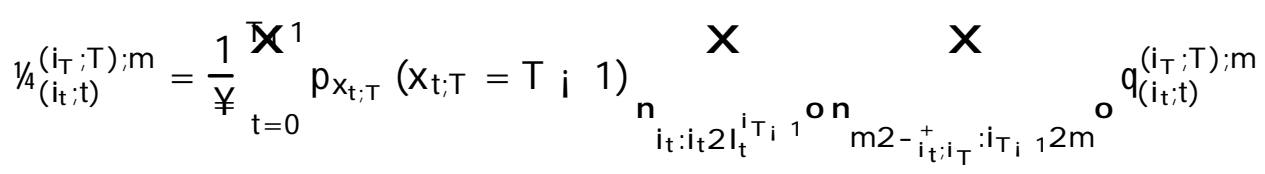

where

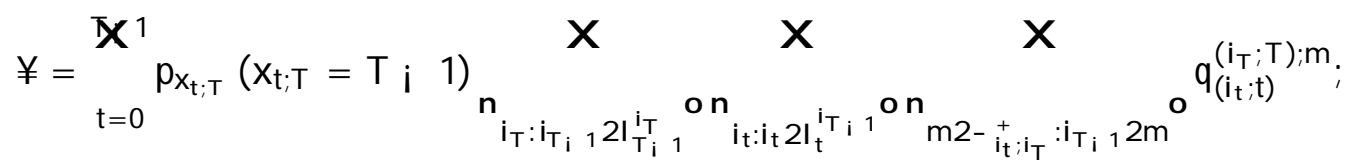




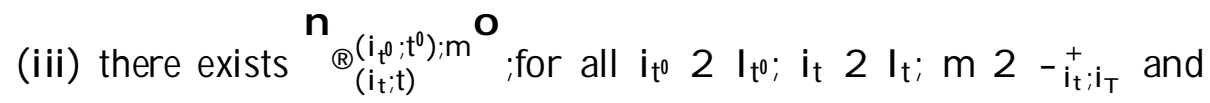
$\mathrm{t}^{0}>\mathrm{t}$ for all $\mathrm{t}=0 ;::: ; \mathrm{T}_{\mathrm{X}} \mathrm{i}$ such that, for all $0<\mathrm{k}<\mathrm{T}$;

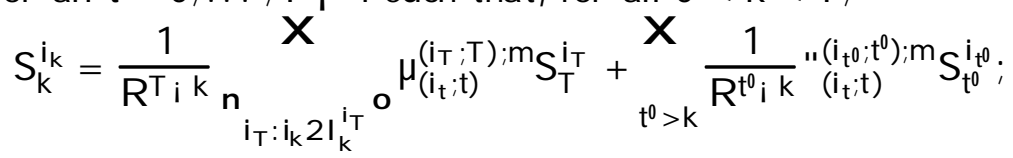

where

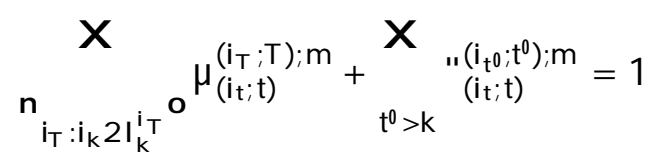

and

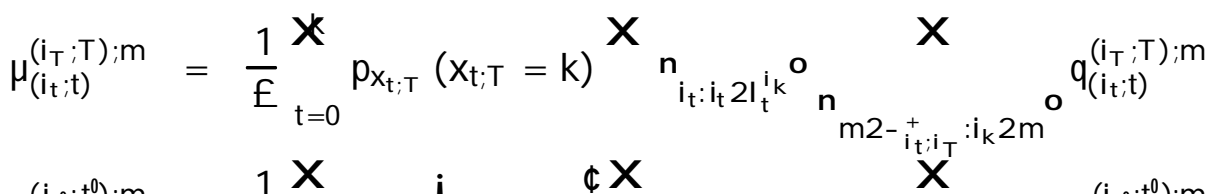

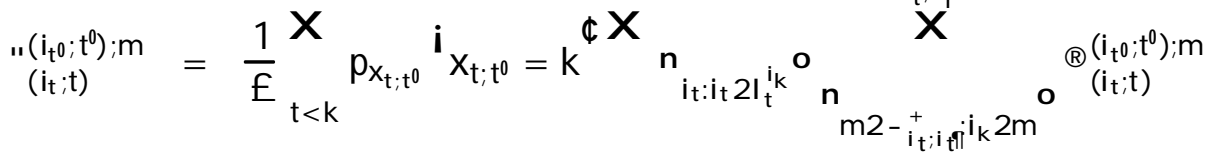

with

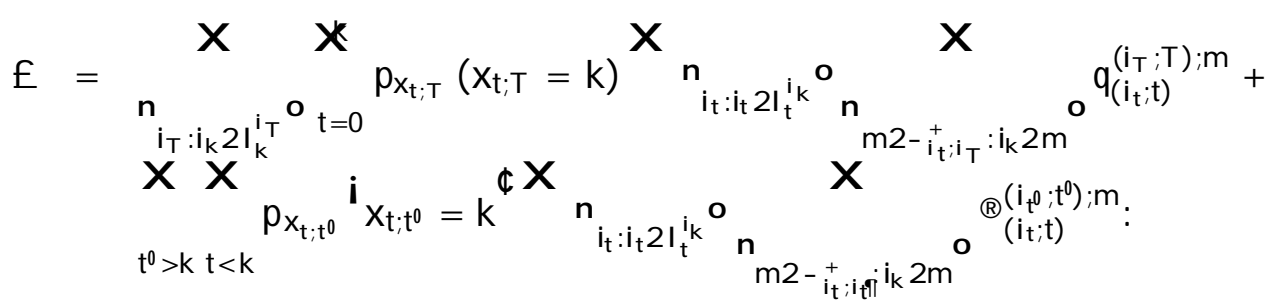

We denote by Qs: the set of modi ${ }^{-}$ed martingale probability measure. Such measures will help writing down the upper and lower bounds for the value of European derivatives under the absence of statistical arbitrage opportunities.

\section{$4 \quad$ Main R esults}

\subsection{The upper bound}

\subsubsection{The Problem}

The problem of determining the upper bound of the statistical arbitrage-free range of variation for the value of a European derivative, can be stated as

$$
\mathrm{C}_{\mathrm{u}}=\min _{\mathrm{f} \mathrm{t}_{\mathrm{t}} ; \mathrm{B}_{\mathrm{t}} \mathrm{g}_{\mathrm{t}=0 ; \ldots ; \mathrm{T}_{\mathrm{i}} 1}} \$ \mathrm{O}_{0}+\mathrm{B}_{0}
$$


where

$$
\$ \mathrm{t} ; \mathrm{B}_{\mathrm{t}} 2 \mathrm{R}^{\mathrm{t}+1} ; \mathrm{t}=0 ; \ldots ; \mathrm{T} ; 1
$$

subject to the conditions of a positive expected payo ${ }^{\circledR}$

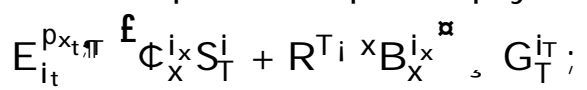

for any $i_{t} 2 I_{t}$ and t $2 f 0 ; 1 ;:: ; T_{i} 1 g^{5}$; and self- nancing conditions

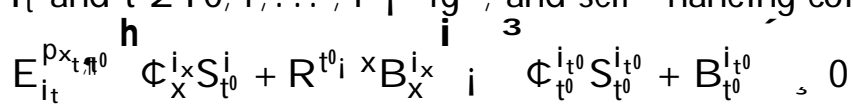

for any $\mathrm{i}_{\mathrm{t}} 2 \mathrm{I}_{\mathrm{t}} ; \mathrm{t}^{0}>\mathrm{t}, \mathrm{t} 2 \mathrm{f} 0 ; 1 ;::: ; \mathrm{T} ; 2 \mathrm{~g}$ and $\mathrm{t}^{0} 2 \mathrm{f} 1 ;::: ; \mathrm{T}$ i $1 \mathrm{~g}^{6}$ :

Example 9 Illustration of the optimization problem with $\mathrm{T}=3$. The evolution of the price underlying asset can be represented by the tree in - gure 1.

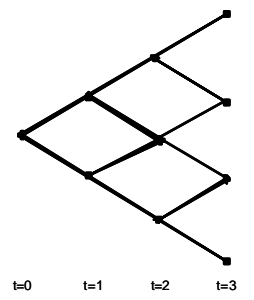

Figure 1: Evolution of the undelying asset' price.

In what concerns the evolution of the price process there are eight di ßerent states, i.e., - $=f w_{i} g_{1}=1 ;:: 8:$ : The problem that must be solved in order to ${ }^{-}$nd the upper bound is the following.

$$
\mathrm{C}_{\mathrm{u}}=\min _{\mathrm{f} \notin \mathrm{t} ; \mathrm{B} \mathrm{Bg}_{\mathrm{t}=0 ; \cdots ; 2}} \phi_{0} \mathrm{~S}_{0}+\mathrm{B}_{0}
$$

where

$$
\begin{aligned}
& f \notin 0 ; B_{0} g=f\left(\$ 0 ; B_{0}\right) g
\end{aligned}
$$

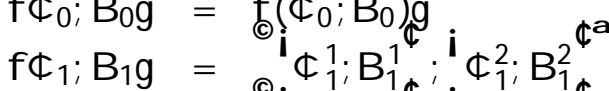

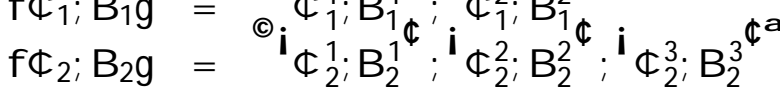

\footnotetext{
${ }^{5}$ For each $i_{t}$ there are $2^{\left(T_{i} t\right)}$ patphs, and as a result, $2^{\left(T_{i} t\right)}(t+1)$ restrictions at time $t$. The total number of restrictions is $\mathrm{Ti}_{\mathrm{t}=0}{ }^{1} 2^{(\mathrm{T} i \mathrm{t})}(\mathrm{t}+1)$ :

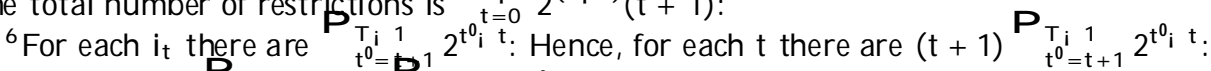

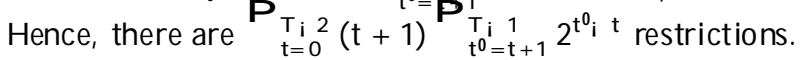


subject to the conditions of a positive expected payo ${ }^{\circledR}$

$$
{ }_{\phi}{ }_{2}^{i_{2}} S_{3}^{i_{3}}+R B_{2}^{i_{2}}, G_{3}^{i_{3}} \text {; }
$$

for all $i_{3} 2 I_{3}$ and $i_{2} 2 I_{2}$ such that $i_{2} 2 I_{2}^{i_{3}}$ : (these are 6 constraints);

$$
p \phi_{2}^{i_{2}} S_{3}^{i_{3}}+R B_{2}^{i_{2}}+(1 i p) \phi_{1}^{i_{1}} S_{3}^{i_{3}}+R^{2} B_{1}^{i_{1}^{i}}, G_{3}^{i_{3}}
$$

for all $i_{3} 2 I_{3} ; i_{2} 2 I_{2}$ and $i_{1} 2 I_{1}$ such that $i_{2} 2 I_{2}^{i_{3}}$ : and $i_{1} 2 I_{1}^{i_{2}} ; i_{\text {i.e., }} i_{1} ; i_{2}$ and $i_{3}$ belong to the same path (these are 8 constraints) and

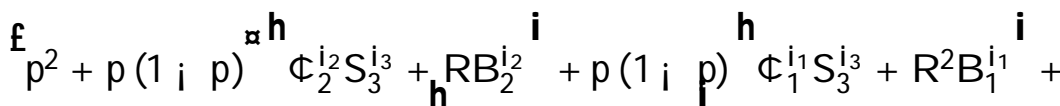

$$
\begin{aligned}
& +(1 ; p)^{2} \$_{0} S_{3}^{i_{3}}+R^{3} B_{2}^{i_{2}}, G_{3}^{i_{3}}
\end{aligned}
$$

for all $i_{3} 2 I_{3} ; i_{2} 2 I_{2}$ and $i_{1} 2 I_{1}$ such that $i_{2} 2 I_{2}^{i_{3}}$ : and $i_{1} 2 I_{1}^{i_{2}} ; i$.e., $i_{1}$; $i_{2}$ and $i_{3}$ belong to the same path (these are 8 constraints). Moreover, the self-" nancing constraints must also be considered

$$
{ }_{0} S_{1}^{i_{1}}+R B_{0}, \phi_{1}^{i_{1}} S_{1}^{i_{1}}+B_{1}^{i_{1}}
$$

for any $i_{1} 2 I_{1}$ ( 2 constraints),

$$
(1 ; \quad p) \stackrel{h}{ } \phi 0 S_{2}^{i_{2}}+R^{2} B_{2}^{i_{2}}+p \phi_{1}^{i_{1}} S_{2}^{i_{2}}+R B_{1}^{i_{1}}, \phi_{2}^{i_{2}} S_{2}^{i_{2}}+B_{2}^{i_{2}}
$$

for any $i_{2} 2 I_{2}$ and $i_{1} 2 I_{1}$ such that $i_{1} 2 I_{1}^{i_{2}}$; $i$.e., $i_{1}$; and $i_{2}$ belong to the same path (these are 4 constraints) and, "nally,

$$
\phi_{1}^{i_{1}} S_{2}^{i_{2}}+R B_{1}^{i_{1}}, \Phi_{2}^{i_{2}} S_{2}^{i_{2}}+B_{2}^{i_{2}}
$$

for any $i_{2} 2 I_{2}$ and $i_{1} 2 I_{1}$ such that $i_{1} 2 I_{1}^{i_{2}}$; i.e., $i_{1}$; and $i_{2}$ belong to the same path (these are 4 constraints).

\subsubsection{Solution}

Theorem 10 There exists a modi ${ }^{-}$ed martingale probability measure, $q^{\top}{ }^{\top} 2$ $\mathrm{Q}_{\mathrm{S}}$; such that the upper bound for arbitrage-free value of a E uropean option can be written as

$$
C_{u}=\max _{q^{i} T 2 Q_{S}}{\frac{1}{R^{\top}}}^{X}{ }_{f i_{T} 2 l_{T} g} q^{i_{\top}} G_{T}^{i_{T}}:
$$




\section{P roof. See proof in appendix A.1.}

Remark 11 If a Call Option is considered, the values for $\mathrm{q}^{\top}$; in a model with two periods are explicitly calculated in appendix A.3. In that case it can be shown that for a strictly positive $p$; the $q^{1}$; $q^{2}$ and $q^{3}$ are also strictly positive.

In what follows we characterize some relevant properties of $\mathrm{C}_{\mathrm{u}}$ :

1. $\mathrm{C}_{\mathrm{u}} \cdot \mathrm{C}_{\mathrm{u}}^{0}$

P roof. Let $\phi_{0}^{0}$ and $\mathrm{B}_{0}^{0}$ denote the optimal values invested, at time $\mathrm{t}=0$; in the stock and in the risk-free asset respectively, when $\mathrm{p}=0$. The trading strategy $\bar{\phi}_{\mathrm{t}}=\stackrel{1}{\leftarrow} \underset{0}{\mathrm{P}}=0$ and $\mathrm{B}_{\mathrm{t}}=\mathrm{R}^{\mathrm{t}} \mathrm{B}_{0}^{\mathrm{p}=0}$, for $\mathrm{t}=1 ; ;: ; ; \mathrm{T}_{\mathrm{i}} 1$, is an admissible strategy for any given $p$. As a result, the solution of the problem for any $p$ cannot be larger that the value of this portfolio at $\mathrm{t}=0$ (which is $\mathrm{C}_{\mathrm{u}}^{0}$ ).

2. $\mathrm{C}_{\mathrm{u}}, \mathrm{C}_{\mathrm{u}}^{1}$ :

P roof. Consider the trading strategy ${ }^{i_{j}}{ }_{t}^{\alpha} ; B_{t}^{\alpha}{ }_{t=0 ;: ;} T_{i}$ i that solves the maximization problem that characterizes the upper bound for a p $2(0 ; 1)$ : This is an admissible strategy for the case $p=1$, because it is self-" nancing, i.e.,

$$
\phi_{t_{i}}^{i_{t_{i}} 1} S_{t}^{i_{t}}+R B_{t_{i} 1}^{i_{t_{i}} 1}, \phi_{t}^{i_{t}} S_{t}^{i_{t}}+B_{t}^{i_{t}}
$$

and superreplicates the payo ${ }^{\circledR}$ of the European derivative at maturity, i.e.,

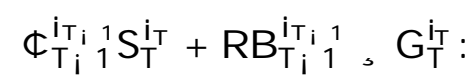

Hence, the solution of the problem for $p=1$ cannot be higher than the value of this portfol io at $t=0$ ( which is $C_{u}$ ).

3. $\lim _{\mathrm{p} !}{ }_{0} \mathrm{C}_{\mathrm{u}}=\mathrm{C}_{\mathrm{u}}^{0}$ and $\lim _{\mathrm{p} !}{ }_{1} \mathrm{C}_{\mathrm{u}}=\mathrm{C}_{\mathrm{u}}^{1}$ :

P roof. See A ppendix A.4

An example for a Call $O$ ption and $T=2$ is also show in appendix A.4.

4. $C_{u}$ is a decreasing function of $p$.

P roof. See A ppendix A.4 
5. For a Call Option and $\mathrm{T}=2$, we can prove that

$$
\mathrm{C}_{\mathrm{u}} \cdot \mathrm{pC}_{\mathrm{u}}^{1}+(1 ; \mathrm{p}) \mathrm{C}_{\mathrm{u}}^{0}
$$

meaning that the probabilistic upper bound is a convex linear combination of the perfectly liquid upper bound and the perfectly illiquid upper bound.

P roof. See appendix A.4.

\subsection{The Lower Bound}

The organization of this section is analogous to the section for the upper bound.

\subsubsection{The Problem}

The problem of determining the lower bound of the statistical arbitrage-free range of variation for the value of an European derivative, can be stated as

$$
C_{l}=\max _{f \notin t ; B t g_{t}=0 ; \cdots ; ; ; 1} \notin 0 S_{0}+B_{0}
$$

where

$$
\phi t ; B_{t} 2 R^{t+1} ; t=0 ; \ldots ; T \text { i } 1
$$

subject to the conditions of a positive expected payo ${ }^{\circledR}$

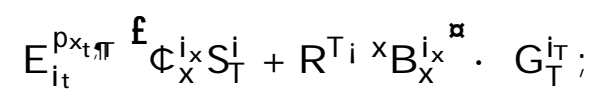

for any $i_{t} 2 I_{t}$ and $t 2 f 0 ; 1 ;::: ; T$ i $1 g^{7}$; and self- ${ }^{-}$nancing conditions

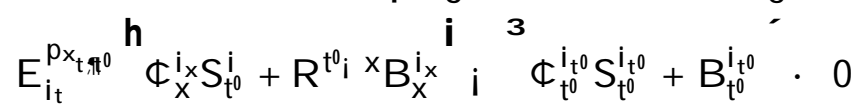

for any $\mathrm{i}_{\mathrm{t}} 2 \mathrm{I}_{\mathrm{t}} ; \mathrm{t}^{0}>\mathrm{t}, \mathrm{t} 2 \mathrm{f} 0 ; 1 ;::: ; \mathrm{T} ; 2 \mathrm{~g}$ and $\mathrm{t}^{0} 2 \mathrm{f} 1 ;::: ; \mathrm{T}$ i $1 \mathrm{~g}^{8}$ :

\footnotetext{
${ }^{7} \mathrm{As}$ in the upper bound case, for each $i_{t}$ there are $2^{\left(T_{i} t\right)}$ paths, and as a result, $2^{(T i t)}(t+1)$ restrictions at timet. The total number of restrictions is $t_{t=0}^{1} 2^{(T i t)}(t+1)$ :

${ }^{8} \mathrm{As}$ in the upper bound case, for each $\mathrm{i}_{\mathrm{t}}$ there are ${ }_{\mathrm{T}^{0}=t_{1 \rightarrow 1}}^{\mathrm{T}^{\mathrm{t}_{i}} \mathrm{t}}$ : Hence, for each $\mathrm{t}$

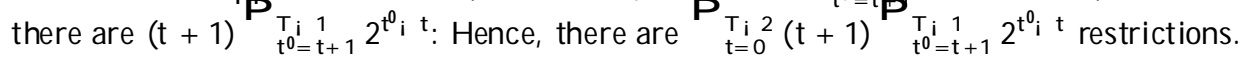




\subsubsection{Solution}

Theorem 12 There exists an modi ${ }^{-}$ed martingale probability measure, $d^{\top}{ }^{2}$ Qs; such that the upper bound for arbitrage free value of an European option can be written as

$$
C_{I}=\min _{q^{i \top} 2 Q_{S}}{\frac{1}{R^{\top}}}^{X} f_{i_{T} 2 I_{T} g} d^{\top \top} G_{T}^{i T}:
$$

P roof. The proof is analogous to the upper bound.

Remark 13 If a call option is considered, the values for $\mathrm{q}^{\top}$; in a model with two periods are explicitly calculated in appendix B.2. In that case it can be shown that for a strictly positive $p$; the $q^{1} ; q^{2}$ and $q^{3}$ are also strictly positive.

In what follows we characterize some relevant properties of $C_{1}$ :

1. $C_{1}, C_{1}^{0}$ :

2. $C_{1} \cdot C_{1}^{1}$ :

3. $\lim _{p !} C_{1}=C_{1}^{0}$ and $\lim _{p !} C_{1}=C_{1}^{1}$ :

An example for a call option and $\mathrm{T}=2$ is shown in appendix ??

4. $C_{1}$ is a increasing function of $p$.

The proofs of these properties are analogous to those presented for the upper bound.

\section{Utility Functions and Reservation Prices}

In this section we show that the price for which any agent is indi ßerent between transacting or not transacting the derivative, to be called the reservation price of the derivative, is contained within the statistical arbitrage bounds derived above.

Let $u_{t}(:)$ denote a utility function representing the preferences of an agent at time $t$. The argument of the utility function is assumed to be the consumption at time $t$. Let $y$ be the initial endowment of the agent, and $Z_{t}$ denote the vector of consumption at time t; i.e., $Z_{t}=Z_{t}^{i_{t}}{ }_{i_{t} 2 I_{t}}$ : Let $1 / 2$ be a discount factor. If an agent sells a European derivative by the amount $\mathrm{C}$; 
and that derivative has a payo ${ }^{\circledR}$ at maturity given $\mathrm{G}_{\mathrm{T}}^{\mathrm{i}_{T}}$, the maximum utility that he or she can attain is

$$
u_{s e l l}^{\alpha}(C ; p)=\sup _{f \notin t ; B t g_{t=0 ; \cdots ; T_{i} 1}} E_{0}^{G ; P}{ }_{t=0}^{T}{ }_{t / 2}^{1 / 2} u_{t}\left(Z_{t}\right)
$$

subject to

$$
\begin{aligned}
& \mathrm{Z}_{0}+\pitchfork \mathrm{S}_{0}+\mathrm{B}_{0} \cdot \mathrm{C}+\mathrm{y}
\end{aligned}
$$

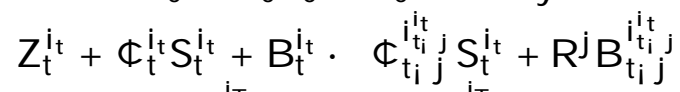

$$
\begin{aligned}
& Z_{T}^{i_{T}} \cdot \phi_{T_{i} j}^{i_{T}^{i_{T}} j} S_{T}^{i_{T}}+R^{j} B_{T_{i} j}^{i_{i}^{i_{T}} j} i G_{T}^{i_{T}}
\end{aligned}
$$

for all $i_{T} 2 I_{T} ; i_{t} 2 I_{t}, j \cdot t$ and $t=1 ;:: ; ; T_{i} 1$ where $E_{0}^{G ; P}$ denotes $a$ bivariate expected value, at $t=0$, with respect to the probability $P$ induced by the market existence and the probability $\mathrm{G}$ underlying the stochastic evolution of the price process.

Similarly, if the agent decides not to include derivatives in his or her portfolio, the maximum utility that he or she can attain is given by

$$
u^{\alpha}(p)=\sup _{f \notin t ; B t g_{t=0 ; \cdots ; T_{i} 1}} E_{0}^{G ; P}{ }_{t=0}^{T}{ }^{1 / 2} u_{t}\left(Z_{t}\right)
$$

subject to

$$
\begin{aligned}
& Z_{0}+\${ }_{0} S_{0}+B_{0} \cdot y \\
& Z_{t}^{i_{t}}+\phi{ }_{t}^{i_{t}} S_{t}^{i_{t}}+B_{t}^{i_{t}} \cdot \phi_{t_{i} j}^{i_{i_{i}}^{i_{t}} j} S_{t}^{i_{t}}+R^{j} B_{t_{i} j}^{i_{t_{i} j}^{i_{t}} t_{j}} \\
& Z_{T}^{i_{T}} \cdot \phi_{T_{i} j}^{i_{T}^{i_{T}}{ }^{j}} S_{T}^{i_{T}}+R^{j} B_{T_{i} j}^{i_{T}^{i_{T}}{ }_{j}}
\end{aligned}
$$

Lemma 14 In the case of random dryness, there is $p^{\alpha}>0$ such that, for all $p<p^{\alpha}$; the utility attained selling the derivative by $C_{u}$, is larger than the utility attained if the derivative is not included in the portfolio.

$$
u_{s e l \mid}^{\alpha}\left(C_{u} ; p\right), u^{\alpha}(p) \text { : }
$$

$P$ roof. Let, for a given $p ; f \notin t ; B_{t} g_{t=0 ;: .: ; T_{i} 1}$ denote the solution of the utility maximization problem with no derivative and $\mathrm{f} \phi \mathrm{t} ; \mathrm{B}_{\mathrm{t}}^{\mathrm{u}} \mathrm{g}_{\mathrm{t}=0 ; \ldots ; \mathrm{T}_{i}}$ de note the solution of the minimization problem that must be solved to ${ }^{-}$nd the upper bound if statistial arbitrage opportunities are considered (see section 4.1). Moreover, let $\phi \underset{t}{s e l l} ; B_{t}^{\text {sel }} t=0 ; \ldots ; T_{i} 1$ denote an admissible solution of the utility maximization problem when the agent sells one unit of 
the derivative. Now, consider the limit case, when $\mathrm{p}$ approaches zero. In that case, the portfolio

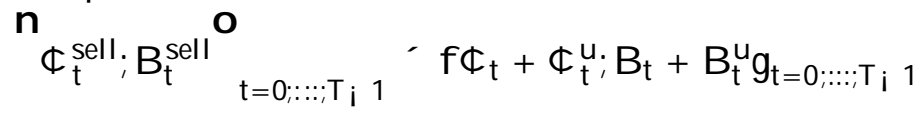

is an admissible solution of the utility maximization problem when the agent sells one unit of derivative by $\mathrm{C}_{\mathrm{u}}$. The reason is as follows. T he constraint set of the problem that must be solved to ${ }^{-}$nd the upper bound is continuous

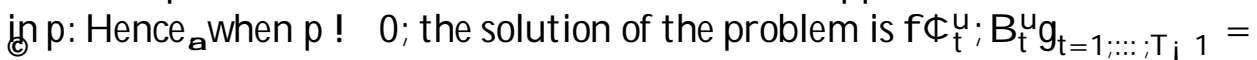
$\phi u_{0} ; R^{t} B_{0}^{u}$ where $f \notin u$; $; B_{0}^{u} g$ is the solution of following problem

$$
\begin{aligned}
& 1 / 2 \quad 3 / 4
\end{aligned}
$$

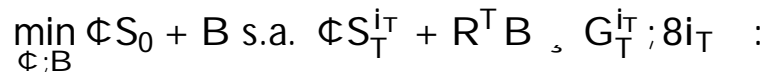

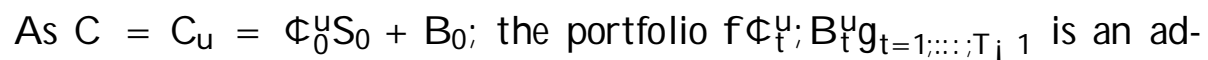
missible solution of the utility maximization problem when one unit of the derivative is being soted. $M$ or eoverer, it guarantees a positive expected utility. ${ }^{9}$ Hence, the portfolio $\phi \mathrm{t}_{\mathrm{t}}^{\text {sell }} ; \mathrm{B}_{\mathrm{t}}^{\text {sell }} \quad \mathrm{t}=0 . \ldots . ; \mathrm{T}_{\mathrm{i}}$ is also admissible solution for the optimization problem, when one unit ' of the derivative is being sold, which

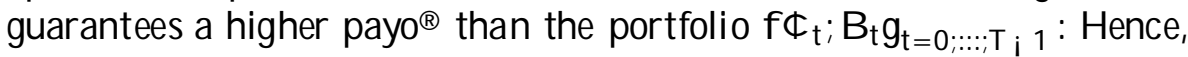

$$
\mathrm{u}_{\mathrm{sell}}^{\infty}\left(\mathrm{C}_{\mathrm{u}} ; 0\right), \mathrm{u}^{\infty}(0) \text { : }
$$

Continuity on $\mathrm{p}$ of both $u_{\mathrm{sell}}^{\mathbb{\alpha}}$ and $\mathrm{u}^{\mathbb{\alpha}}$ ensure the result.

Remark 15 Notice that the existence of $p^{\alpha}$ follows from the continuity of the utilities in p: Furthermore, it is possible to have $p^{\alpha}=1$. Examples with di ßerent values of $p^{\alpha}$ are given in the end of this paper. The range of values $p<p^{\alpha}$ characterizes what was vaguely described as $\backslash$ su \pm ciently incomplete markets" in the introduction.

The reservation price for an agent that is selling the option is de ned as the value of $C$ that makes $u_{s e l \mid}^{\alpha}(C ; p)=u^{\alpha}(p)$. Let $R_{u}$ denote such reservation price.

Theorem 16 For all $p<p^{\alpha}$ we have $R_{u} \cdot C_{u}$ :

P roof. The optimal utility value;

$$
u_{s e l l}^{\alpha}(C ; p)=\sup _{f \phi_{t} ; B_{t} g_{t=0 ;: ; ; i 1}} C+y_{i}\left(\phi S_{0} S_{0}+B_{0}\right)+E_{0}^{G ; P}{ }_{t=1}^{T}{ }_{t / 2} u_{t}\left(Z_{t}\right) ;
$$

\footnotetext{
${ }^{9}$ It su \pm ces to consider $Z_{0}=y ; Z_{i t}=0$ and $Z_{i_{T}}=\phi S_{T}^{i_{T}}+R^{\top} B i G_{T}^{i_{T}}, 0$ :
} 
is increasing in C: This, together with lemma 14 leads to the result.

The same applies for the case when the agent is buying a derivative. In that case, if an agent is buying the derivative by $\mathrm{C}$, the maximum utility that he or she can attain is

$$
u_{\text {buy }}^{\alpha}(C ; p)=\sup _{f \phi_{t} ; B_{t} g_{t=0 ; \ldots ; T_{i} 1}} E_{0}^{G ; P}{ }_{t=0}^{T}{ }_{t / 2 u_{t}}\left(Z_{t}\right)
$$

subject to

$$
\begin{aligned}
& \mathrm{Z}_{0}+\${ }_{0} \mathrm{~S}_{0}+\mathrm{B}_{0} \cdot \quad \mathrm{i} \mathrm{C}+\mathrm{y}
\end{aligned}
$$

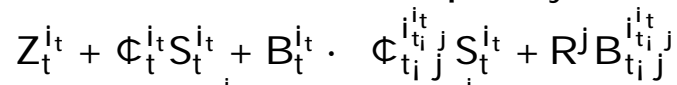

$$
\begin{aligned}
& Z_{T}^{i_{T}} \cdot \phi_{T_{i} j}^{i_{T}^{i_{T}} j} S_{T}^{i_{T}}+R^{j} B_{T_{i} j}^{i_{T_{T} j}^{i_{j}}}+G_{T}^{i_{T}}
\end{aligned}
$$

Lemma 17 In the case of random dryness, there is $p^{\alpha}>0$ such that, for all $p<p^{\alpha}$; the utility attained buying the derivative by $C_{1}$, is larger than the utility attained if the derivative is not included in the portfolio.

$$
u_{\text {buy }}^{\infty}\left(C_{1} ; p\right), u^{\alpha}(p) \text { : }
$$

$P$ roof. The proof is analogous to the one in proposition 14

Let $R_{l}$ denote the reservation selling price, i.e., the price such that $u^{\alpha}(p)=u_{\text {buy }}^{\alpha}\left(R_{1} ; p\right)$.

Theorem 18 For all $p<p^{\alpha}$ we have $R_{I}, C_{l}^{p}$ :

P roof. The proof is analogous to the one presented in theorem (16). However, in this case the utility is a decreasing function of $C$; and we obtain

$$
\left.u_{\text {buy }}^{\infty}\left(C_{1} ; p\right), u^{\alpha}(p)\right) R_{1}, C_{1}:
$$

Several illustrations are presented in section 7.

\section{Comparisons with the Literature}

In what follows we compare our methodology with others in the literature, namely Bernardo and Ledoit (2000) and Cochrane and Sa\$-R equejo (2000). Cochrane and Sa\$-R equejo (2000) introduce the notion of Igood deals", or investment opportunities with high Sharpe ratios. They show that ruling out investment opportunities with high Sharpe ratios, they can obtain narrower 
bounds on securities prices. However, as stressed in Bondarenko (2003), not all pure arbitrage opportunities qualify as Igood deals". Moreover, for a given set of parameters we found out that in order to contain the reservation prices of a risk neutral agent the interval is more broad than the one that was obtained in our formulation.

We ${ }^{-}$rst provide a simple example to compare our bounds with pure arbitrage bounds.

Example 19 Consider a simple two periods example, where transactions are certainly possible at times $t=0$ and $t=2$ : At time $t=1$ there are transactions with a given probability $p=0: 65$ : The initial stock price is $\mathrm{S}_{0}=100$ and it may either increase in each period with a probability $0: 55$; or decrease with a probability $0: 45$ : We take and $U=1: 2 ; D=0: 8$ and $R=1: 1$ : A call option that matures at time $T=2$ with exercise price $K=80$ is considered. Using pure arbitrage arguments we ${ }^{-}$nd the following range of variation for the value of the call option

[33:88; 37:69]

Using the notion of statistical arbitrage opportunity, the above range gets narrower and is given by

$$
\text { [34:31; 35:17]; }
$$

clearly narrower that the above interval.

If markets were complete $(p=1)$, the value of the option would be 34:71. Also, the reservation price ${ }^{10}$ for a risk neutral agent is equal to 35:09. Notice that both intervals include the complete market value and the reservation price.

We now use the same example to compare our methodology with the one presented by Cochrane and Sa $\$$-R equejo (2000). We show that either our interval is contained in theirs, or else, their interval do not contain the above mentioned reservation price.

W ith the Sharpe ratio methodology the lower bound is given by

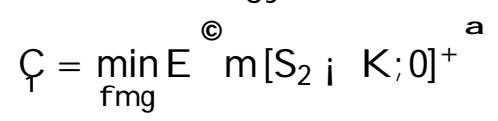

\footnotetext{
${ }^{10}$ In this example, the reservation price for an agent who is buying the derivative coincides with that of an agent who is selling it.
} 
subject to

$$
\left.\mathrm{S}_{0}=\mathrm{E}\left[\mathrm{mS}_{2}\right] ; \mathrm{m}, 0 ; 3 / 4 \mathrm{~m}\right) \cdot \frac{\mathrm{h}}{\mathrm{R}^{2}} ;
$$

where $\mathrm{S}_{0}$ is the initial price of the risky asset, and $\mathrm{S}_{2}$ is the price of the risky asset at time $t=2^{11}$ : The upper bound is

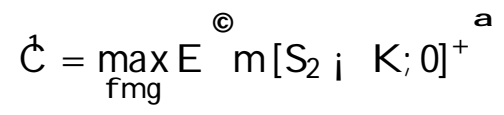

subject to

$$
\left.\mathrm{p}=\mathrm{E}\left[\mathrm{mS}_{2}\right] ; \mathrm{m}, 0 ; 3 / 4 \mathrm{~m}\right) \cdot \frac{\mathrm{h}}{\mathrm{R}^{2}}:
$$

Example 20 In order to compare the statistical arbitrage interval with the Sharpe ratio bounds, we must choose the ad-hoc factor $\mathrm{h}$ so as to make one of the limiting bounds to coincide. If we want the upper bound of the Sharpe Ratio methodology to coincide with the upper bound obtained with statistical arbitrage, we must take $h=0: 3173$ : In that case, the lower bound will be 33:88 and the range of variation will be

\section{[33:88; 35:17];}

worse than the statistical arbitrage interval.

A Iternatively, if we want the lower bound of the Sharpe Ratio methodology to coincide with the lower bound obtained with statistical arbitrage, we must take $h=0: 28359: 12$ In that case, the upper bound will be 34:49 and the range of variation will be

$$
\text { [34:31; 34:49]: }
$$

Although this interval is tighter than the statistical arbitrage interval, it does not contain the reservation price for a risk neutral agent.

In a di ßerent paper Bernardo and Ledoit (2000) preclude investments o Rering high gain-loss ratios to a benchmark investor, somehow analogous

\footnotetext{
${ }^{11}$ A s stressed by Cochraneand Sa $\$$-R equejo, in a former paper Hansen and J agannathan (1991) have shown that a constraint on the discount factor vol atility is equivalent to impose an upper limit on the Sharpe ratio of mean excess return to standard deviation.

${ }^{12}$ In order to get a lower bound higher than 33:88 it is necessary to impose aditionally that $m>0$ : If that were not the case, then the lower bound would only be de- ned for $h$ , 0:2980 and would be equal to 33:88:
} 
to the Igood deals" of Cochrane and S\$a-R equejo. The criterion, however, is di ßerent since Bernardo and Ledoit (2000) propose a utility-based approach, as stressed in the Introduction. In this way, the arbitrage-free range of variation for the value of the European derivative is narrower than in the case of pure arbitrage. Let $z^{+}$denote the (random) gain and $z^{i}$ denote the (random) loss of a given investment opportunity. The utility of a benchmark agent characterizes a pricing kernel that induces a probability measure, according to which the expected gain-loss ratio is bounded from above

$$
\frac{E\left(z^{+}\right)}{E\left(z^{i}\right)} \cdot L^{1}:
$$

The fair price is the one that makes the net result of the investment to be null. In other words, for a benchmark investor, it would correspond to the pricing kernel that would make

$$
E^{i} z^{+} i z^{\Phi}=0, \frac{E\left(z^{+}\right)}{E\left(z^{i}\right)}=1:
$$

This last equality characterizes the benchmark pricing kernel for a given utility.

Notice that the fair price constructed in this way coincides with our de $e^{-}$ition of the reservation price. Therefore, by choosing $\mathbb{E}^{\mathrm{B}}$ larger than one, the interval built by Bernardo and Ledoit contains by constr uction the reservation price of the benchmark agent.

On the other hand, the arbitrary threshold $\mathbb{Z}$ can be chosen such that their interval is contained in the statistical arbitragefree interval.

The disadvantages, however, are clear. First, the threshold is ad-hoc, just as in the case of Cochrane and S\&a-R equejo; second, the constructed interval depends on the benchmark investor; and " nally, the only reservation price that is contained for sure in that interval, is the reservation price of the benchmark investor. In other words, we cannot guarantee that the reservation price of an arbitrary agent, di ßerent from the benchmark, is contained in that interval.

\section{Numerical Examples}

\subsection{Upper and Lower Bounds}

In this section several numerical examples are provided in order to illustrate the properties of the upper and lower bounds presented in the previous 
sections.

Using numerical examples we can conclude that, for a call option,

$$
\mathrm{C}_{\mathrm{u}} \cdot \mathrm{pC}_{\mathrm{u}}^{1}+\left(1_{\mathrm{i}} \quad \mathrm{p}\right) \mathrm{C}_{\mathrm{u}}^{0} \text { : }
$$

If the Call Option is sold by the expected value of the call, regarding the existence (or not) of market, there will be an arbitrage opportunity in statistical terms. The reason is that the agent that sells the call option can buy a hedging portfolio (in a statistical sense) by an amount smaller than the expected value of the call option. As a result, there is an arbitrage opportunity, because he is receiving more for the call option than is paying for the hedging portfolio.

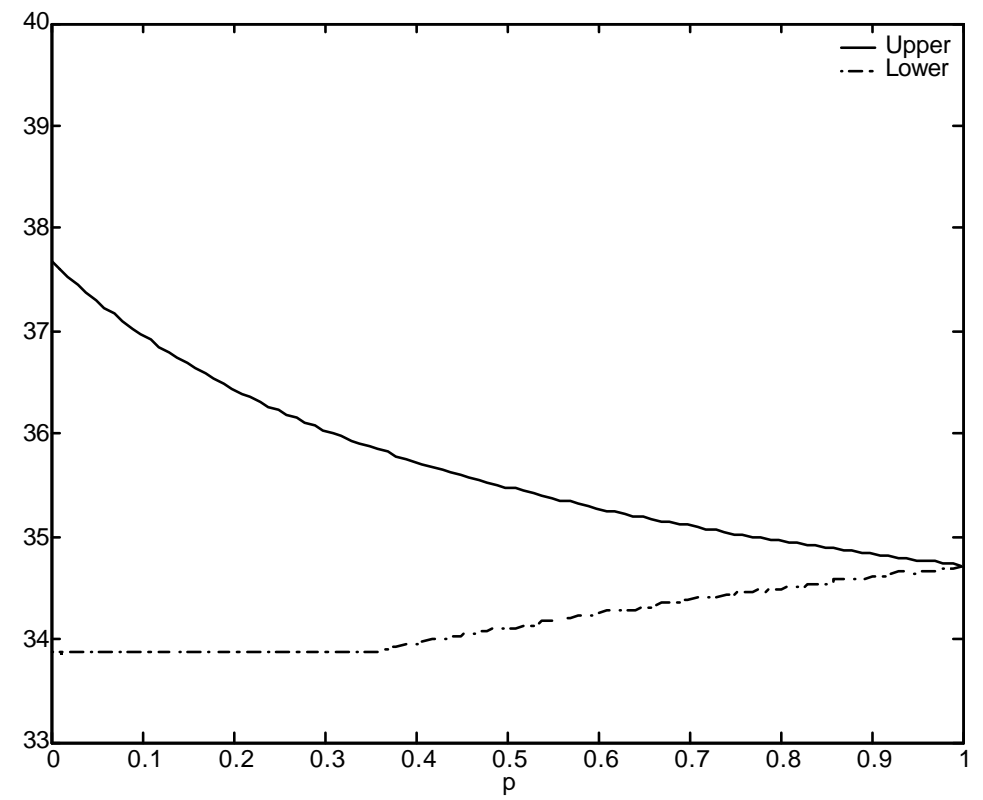

Figure 2: The Upper and Lower B ounds for a E uropean Call Option with di Rerent value of $p$ in a two period model, with $U=1: 2, R=1: 1, D=0: 8$; $\mathrm{S}_{0}=100$ and $\mathrm{K}=80$ :

However, in what concerns the lower bound, it is not possible to conclude whether $C_{1} \cdot \quad p C_{1}^{1}+(1 ; p) C_{1}^{0}$ or $C_{1}, \quad p C_{1}^{1}+(1 ; p) C_{1}^{0}$ : That depends on the value of the parameters. Although in the two-period simulation in Figure 2 we seem to have the former case, the three period example in F igure 3 seems to suggest the latter, since the lower bound behaves as a concave function of $p$ for most of its domain. 


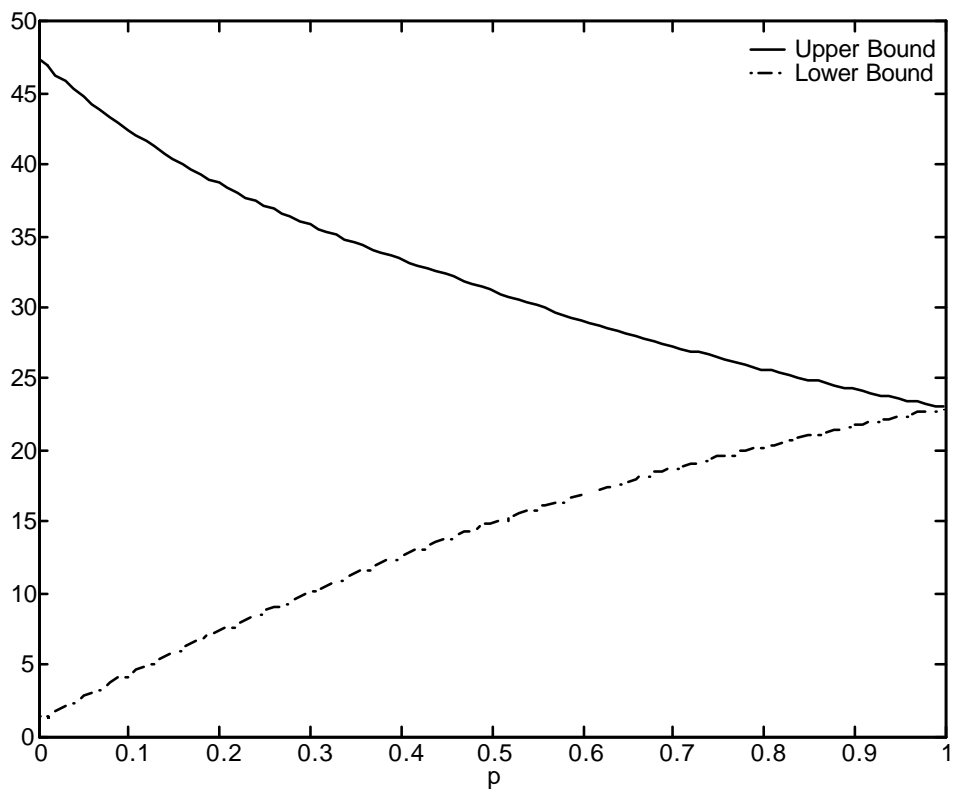

Figure 3: The Upper and Lower Bounds for a E uropean Call Option with di ßerent value of $p$ in a thre period mode, with $U=1: 3, R=1, D=0: 6$; $\mathrm{S}_{0}=100$ and $\mathrm{K}=100$ :

Finally, we may use Figure 4 to illustrate several features.

The Upper and Lower bounds of an European Call Option for di Rerent values of $p$ and $K(K=80, K=100, K=120, K=140$ e $K=160)$ in $a$ three period model, with $U=1: 2 ; R=1: 1 ; D=0: 8$ and $S=100$.

First, let us regard the situations in this $\mathrm{F}$ igure that are related to pure arbitrage. This includes the value of the derivative under perfectly liquid $(p=1)$ and perfectly illiquid $(p=0)$ markets. In the former case, the unique value of the derivative clearly decreases with the exercise price $K$; as it should. In the latter, both the upper bound $C_{u}^{0}$ and the lower bound $C_{1}^{0}$ also decrease with $\mathrm{K}$ : More curiously, however, the spread $\mathrm{C}_{\mathrm{u}}^{0} \mathrm{i} \mathrm{C}_{1}^{0}$ has a non-monotonic behaviour. Obviously this di ßerence is null for $\mathrm{K}$ less than the in ${ }^{-}$mum value of the stock at maturity and must go to zero as the strike approaches the supremum of the stock's possible values at maturity. In the middle of this range it attains a maximum. In our numerical example we observe that the maximum value of the spread is attained for $\mathrm{K}$ close to 120. 


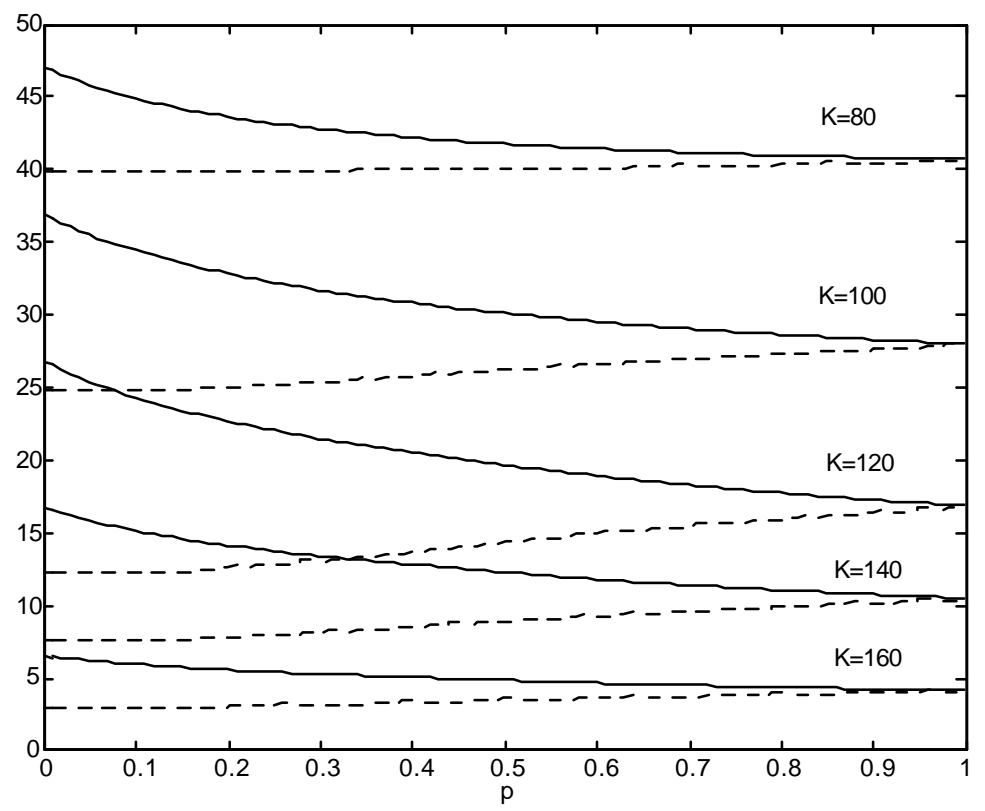

Figure 4: The Upper and Lower bounds of an European Call Option for di ßerent values of $\mathrm{p}$ and $\mathrm{K}(\mathrm{K}=80, \mathrm{~K}=100, \mathrm{~K}=120, \mathrm{~K}=140 \mathrm{e}$ $K=160$ ) in a three period model, with $U=1: 2 ; R=1: 1 ; D=0: 8$ and $\mathrm{S}=100$.

Regarding the statistical arbitrage domain when p $2(0 ; 1)$; we notice that all the above remarks remain true. Figure 4 also suggests that, for any given $p$; the spread attains its maximum for the same value of $K$ as before. Notice that the spread $C_{u}$ i $C_{l}$ decreases with $p$ for ${ }^{-}$xed strike $K$ converging to zero as p ! 1: Hence, although somehow di ßerent from the traditional de nition of arbitrage, the notion of statistical arbitrage seems to provide a very nice bridge, for $0<p<1$, between the two extreme cases above ( $p=0$ and $p=1$ ), where the original concept of arbitrage makes sense. This is illustrated in a more direct way in Figure 5.

A third issue driven by Figure 4 is that coexisting derivatives may restrict more the no-arbitrage interval. As an example, suppose that derivatives with $\mathrm{K}=120$ and $\mathrm{K}=140$ coexist. When $\mathrm{p}=0$, the upper bound for $\mathrm{K}=140$ is abovethe lower bound when $\mathrm{K}=120$. In order to avoid arbitrage opp ortunities, the equilibrium selling price of the $K=120$ derivative has to be above the equilibrium buying price of the $K=140$ derivative. Otherwise 


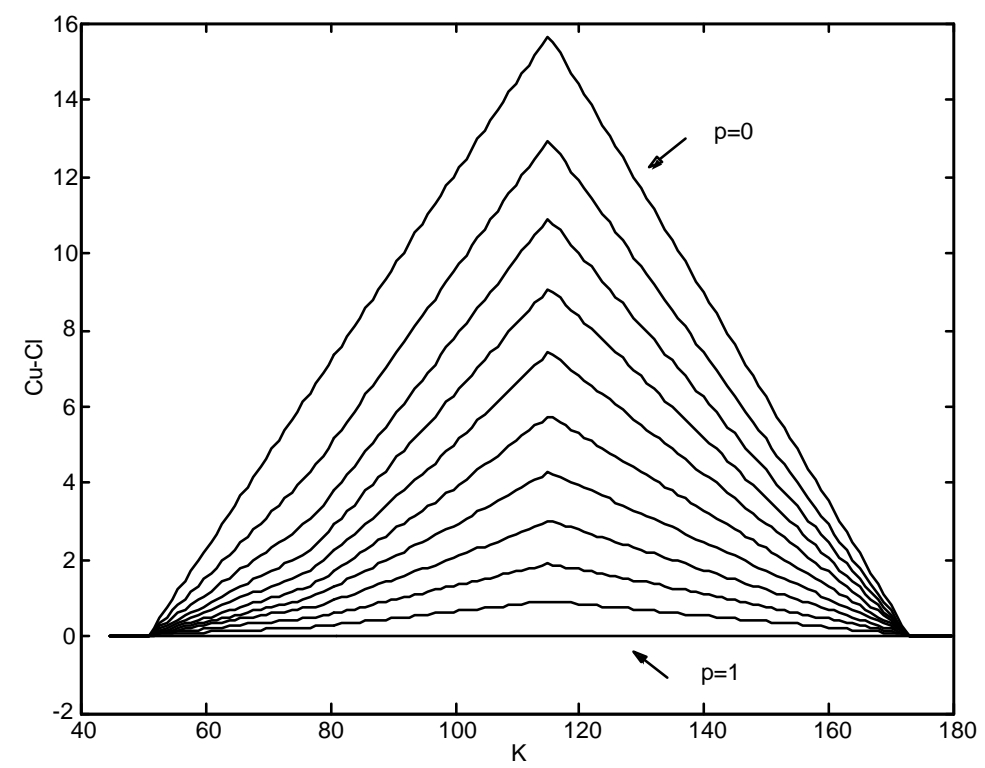

Figure 5: The Spread $\left(C_{u}\right.$ i $\left.C_{l}\right)$ of an European Call Option for di Rerent values of $K$ and $p(p=0 ;:: ; ; 1$ with increments of $0: 1)$ in a three period model, with $U=1: 2 ; R=1: 1 ; D=0: 8$ and $S=100$.

one would buy the $K=120$ derivative with the proceeds of selling the $\mathrm{K}=140$ derivative, to get a net positive payo ${ }^{\circledR}$ at maturity at no cost. This implies that the upper bound of the $\mathrm{K}=140$ derivative should be the equilibrium selling price of the $K=120$ derivative and not necessarily the bound we derived above. This situation stresses the limitation that our bounds were constructed assuming that there was only one derivative that, aditionally, was not part of the replicating portfolio. In fact, the presence of more derivatives may help to complete the market, making the overlapping arbitrage-free regions not viable. As markets become complete, the arbitrage-free regions shrink to a point, corresponding to the unique value of the derivatives under complete markets.

\subsection{Utility and Reservation Prices}

In this section we illustrate several aspects related to the determination of the reservation price. In Figure 6 we represent the utility of an agent in three di Rerent situations. Without the derivative; a short position on the 
derivative, when the instrument is sold by the statistical arbitrage upper bound; and a long position on the derivative when the instrument is bought by the statistical arbitrage lower bound. Notice that for $p=0$ the best situation is the short position on the derivative and the worst is without trading the derivative. This is consistent with lemma 14 and lemma 17. Notice that there is a value of $p$ such that, for larger probabilities, the utility without trading the derivative is no longer the worst. That critical value of $p$ is what we called $p^{\alpha}$ :

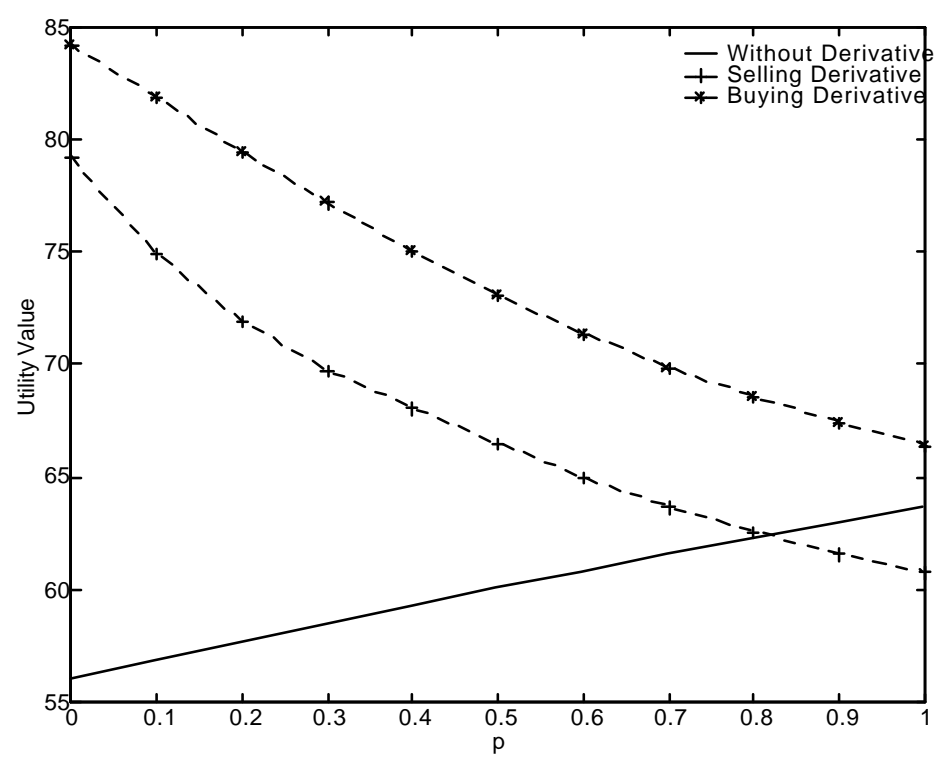

Figure 6: Utility value for the following parameters: $U=1: 3, D=0: 6$, $R=1, S_{0}=100, K=100,1 / 2=1=R, q=0: 5, y=50$ and $T=3$.

Figure 7 represents the statistical arbitrage-free interval together with the reservation price for a risk neutral agent. By construction, the probability associated to the point where the reservation price coincides with the upper bound, corresponds to the critical probability $p^{\alpha}$ : Notice from Figure 6 that the utility of the position associated to a long position on the derivative is always above the utility without trading the derivative. This implies that the reservation price is always above the lower bound. Likewise, the fact that the utility of the short position on the derivative goes below the utility without trading the derivative, implies that the reservation price goes above the upper bound. 


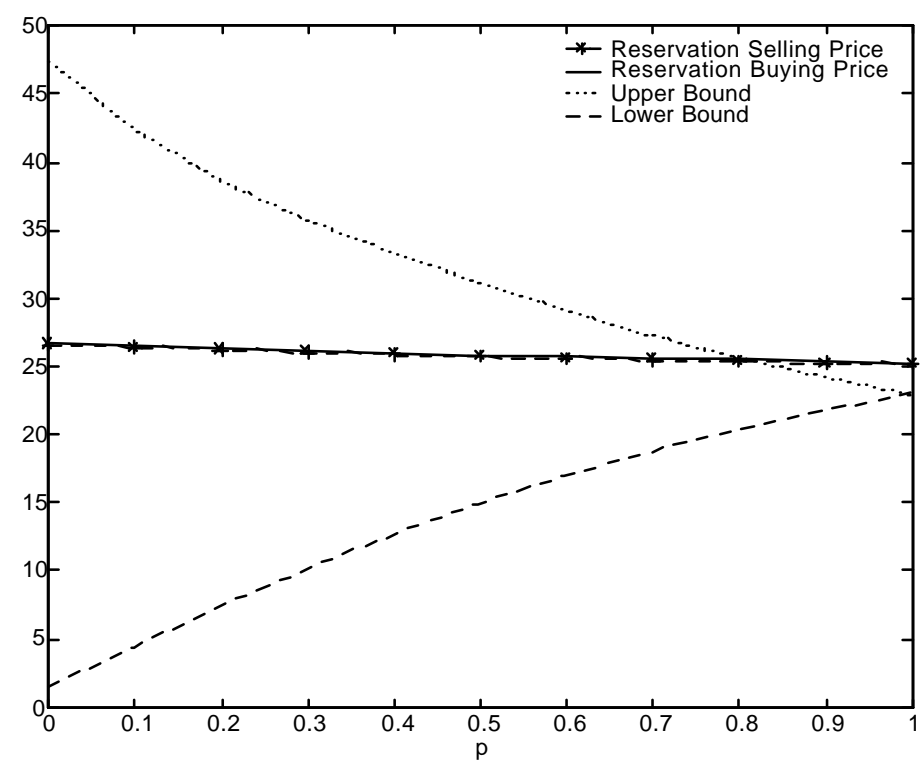

Figure 7: Statistical A rbitrage free bounds and reservation prices for the following parameters: $U=1: 3, D=0: 6, R=1, S_{0}=100, K=100$, $1 / 2=1=F, q=0: 5, y=40$ and $T=3$. 


\section{Conclusion}

In this paper we have characterized the statistical arbitrage-free bounds for the value of an option written on an asset that may not be transacted. This statistical arbitrage-free interval is by construction tighter than the usual arbitrage-free interval, obtained under the superreplication strategy. In that sense, our result is close to the results of Bernardo and Ledoit (2000) and Cochrane and Saq-R equejo (2000). B y using a concept of statistical arbitrage, in the spirit of Bondarenko's (2003), we were able to avoid the arbitrary threshold that led the former approaches to constrain the arbitrage-free interval.

In a framework characterized by the fact that transactions of the underlying asset are possible with a given probability, we derived the range of variation for the statistical arbitrage-free value of an European derivative. If transactions were possible at all points in time there would be a unique arbitrage free value for the E uropean derivative that is contained in the statistical arbitrage-free range. Moreover, the statistical arbitrage-free range is contained in the arbitragefree range of variation if the market is perfectly illiquid. The upper bound is a decreasing function in the probability of existence of the market and the lower bound is a increasing function. They are asymptotically well behaved both when $p$ ! 0 and when $p$ ! 1 .

Finally, we could also prove that, in the case of random illiquidity, the reservation prices (both for selling and buying positions) are contained in the statistical arbitrage free range of variation for the value of the European Option. 


\section{R eferences}

[1] A maro de Matos, J . and P. A ntao, 2001, I Super-Replicating B ounds on European Option Prices when the Underlying A sset is Illiquid", Economics Bulletin, 7, 1-7.

[2] Bernardo, A. and Ledoit, O., 2000, Gain, Loss, and Asset Pricing, J ournal of Political Economy, 108, 1, 144-172.

[3] Bondarenko, O., 2003, Statistical A rbitrage and Securities Prices, Review of F inancial Studies, 16, 875-919.

[4] Cochrane, J . and Sa\$-R equej 0, J ., 2000, B eyond A rbitrage: Good-Deal A sset Price Bounds in Incomplete Markets, J ournal of Political Economy, 108, 1, 79-119.

[5] Edirisinghe, C., V. Naik and R. Uppal, 1993, Optimal Replication of Options with Transaction Costs and Trading Restrictions, J ournal of Financial and Quantitative A nalysis, 28, 117-138.

[6] El Karoui, N. and M.C. Quenez, 1991, Programation Dynamique et Qvaluation des actifs contingents en march incomplet, Comptes Rendues de I' Academy des Sciences de Paris, S\$rie I, 313, 851-854

[7] El Karoui, N. and M.C. Quenez, 1995, Dynamic programming and pricing of contingent claims in an incomplete market, SIAM J ournal of Control and O ptimization, 33, 29-66.

[8] Hansen. L.P. and R. J agannathan. I mplications of Security Market Data for Models of Dynamic Economies. J ournal of Political Economy, 99, 225-262, 1991.

[9] Karatzas, I. and S. G. Kou, 1996, On the Pricing of Contingent Claims with Constrained Portfolios, A nnals of Applied Probability, 6, 321-369.

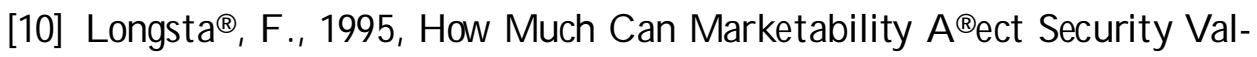
ues?, The J ournal of Finance, 50, 1767\{1774.

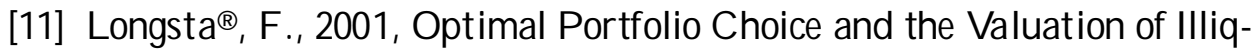
uid Securities, Review of Financial Studies, 14, 407-431.

[12] Longsta $\AA_{1}$ F ., 2004, T he F light-to-Liquidity Premium in U.S. Treasury Bond Prices, J ournal of Business, 77, 3.

[13] Mas-Colell, A., Whiston, M. and Green, J ., 1995, Microeconomic Theory, Oxford University Press. 


\section{A Some Proofs on the Solution of the U pper B ound for Statistical A rbitrage Opportunities}

\section{A.1 Proof of theorem 10}

Proof. For any given path $\mathrm{m} 2-{ }_{i_{t} ; i_{T}}^{+} l e t, \quad\left(i_{T} ; T\right) ; m$ be the dual variable associated with the superreplication constraint

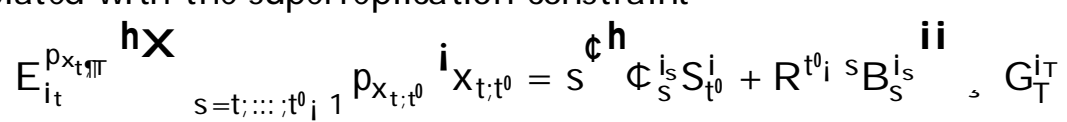

with $\mathrm{i}_{\mathrm{k}} 2 \mathrm{I}_{\mathrm{k}}^{\mathrm{i}_{\mathrm{k}+1}}$ and $\mathrm{k}=\mathrm{t} ;::: ; \mathrm{T} \mathrm{i} 1$ : Let $\mathrm{n}_{\mathrm{t}}^{\mathrm{i}_{\mathrm{T}}}$ be the number of nodes that are predecessors of node $i_{T}$ at time t; where $n_{t}^{i_{T}}$ is given by

$$
\mathrm{n}_{\mathrm{t}}^{\mathrm{i} \mathrm{T}}=\operatorname{minf} \mathrm{T}_{\mathrm{i}}\left(\mathrm{i}_{\mathrm{T}} \mathrm{i} \quad 1\right) ; \mathrm{i}_{\mathrm{T}} \mathrm{i} \quad 1 ; \mathrm{T}_{\mathrm{i}} \operatorname{tg}+\frac{1}{3}
$$

At each node $i_{t}$; that is a predecessor of $i_{T}$; there are \# $-{ }_{i_{t} ; i_{T}}^{+}$paths to reach $i_{T_{T}}$ : For any given path $\mathrm{m} 2-\underset{i_{t} ; i_{0} 0}{+}$ let $\mathbb{B}_{\left(i_{t} ; t\right)}^{\left(i_{0} ; t+t\right) ; m}$ be the dual variable associated with the self-" nancing constraints

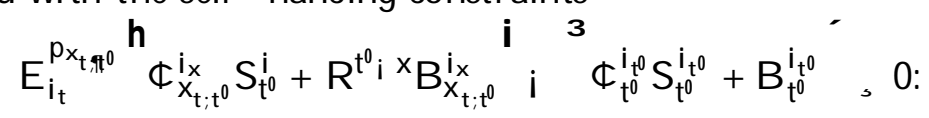

Considering $n_{t}^{i_{t} 0}$ be the number of nodes that are predecessors of node $i_{t} 0$ at time t we have

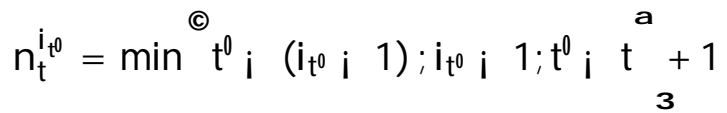

At each node $i_{t}$ that is a predecessor of $i_{t^{0}}$ there are \# $-{ }_{i_{t} ; i_{t} 0}^{+}$.

The problem that must be solved in order to " nd the upper bound of the range of variation of the arbitrage free value of an $E$ uropean derivative is a linear programming problem. Its dual problem is

$$
\min _{{ }_{i_{T}}{ }_{j=1}{ }^{+1},{ }^{i_{T}} G_{T}^{i_{T}}}
$$

where, ${ }^{i_{T}}$ is the sum of the dual variables associated with the positive expected payo ${ }^{\circledR}$ constraints that have the right member equal to $\mathrm{G}_{\mathrm{T}}^{\mathrm{i}_{\mathrm{T}}}$; i.e.,

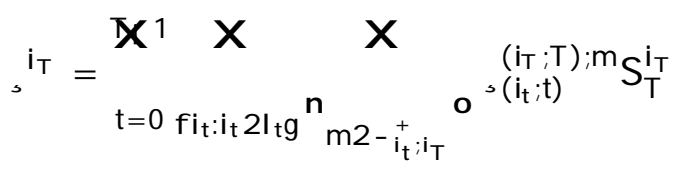


The ${ }^{-}$rst set of constraints is of nonnegativity of each dual variable, i.e,

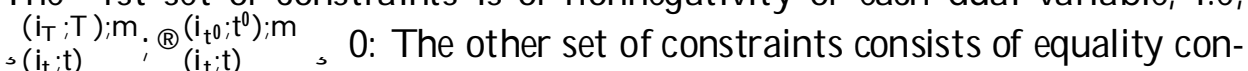
straints, one constraint associated with each variable of the primal problem.

As there are

$$
2^{\mathrm{X}=0} \mathrm{Ti}_{\mathrm{t}=0}(\mathrm{t}+1)=2 \frac{1+\left(\mathrm{T}_{\mathrm{i}} 1+1\right)}{2} \mathrm{~T}=\mathrm{T}(\mathrm{T}+1)
$$

primal variables there are also $\mathrm{T}(\mathrm{T}+1)$ constraints of the dual problem, which are equality constraints because the variables of the primal problem are free.

The constraint for $₫$ o is:

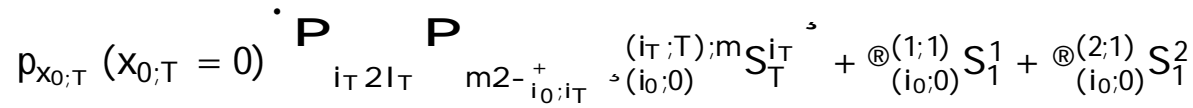

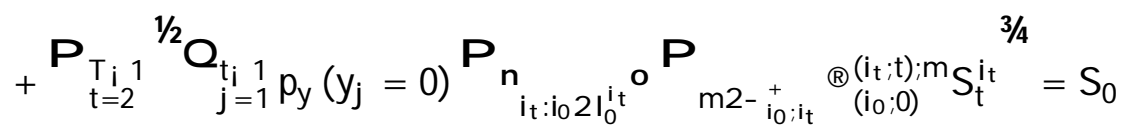

The constraint for $B_{0}$ is:

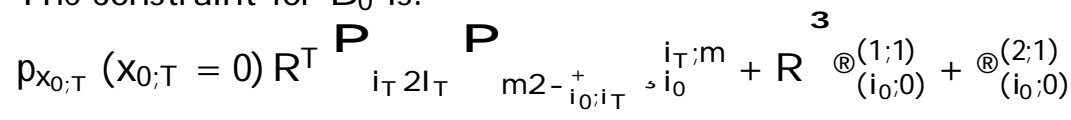

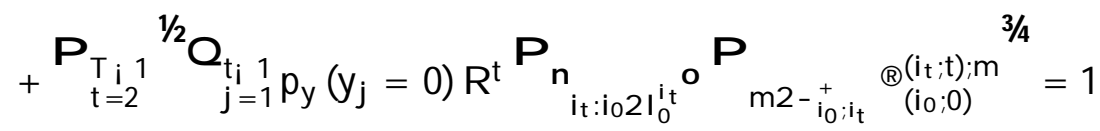

For the constraint that concerns $\phi_{i_{k}}$ the term in, is

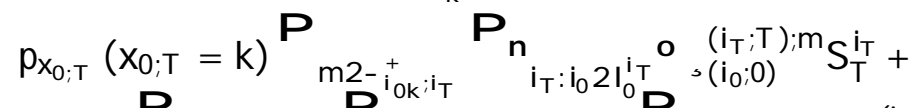

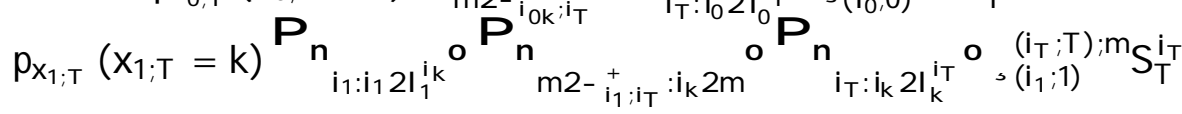

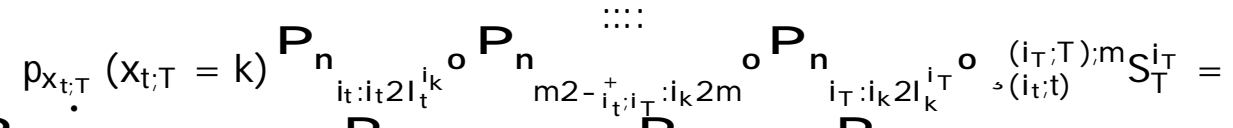

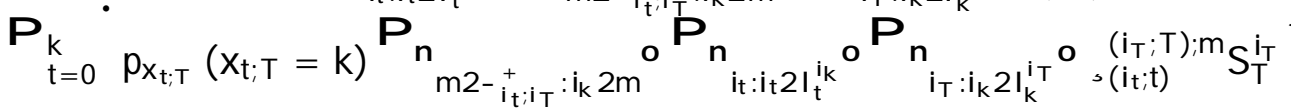

The terms that involve ${ }^{\circledR}$ are

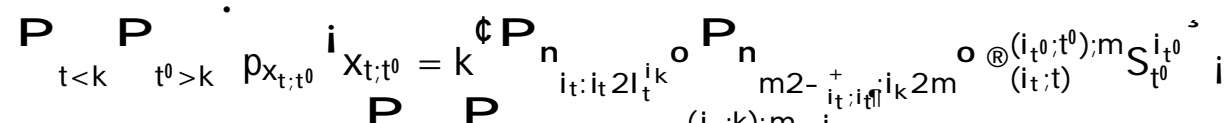

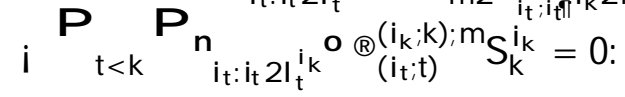


Hence, the constraint for $\phi_{\mathrm{i}_{\mathrm{k}}}$ is

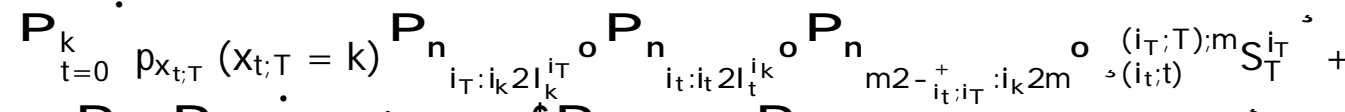

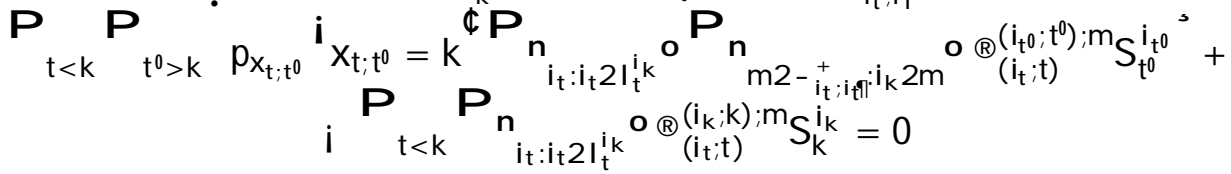

The constraint for $B_{i_{k}}$ is:

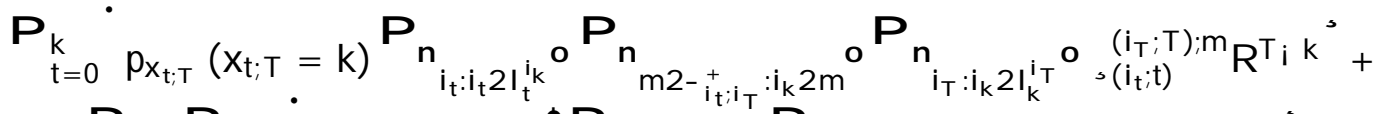

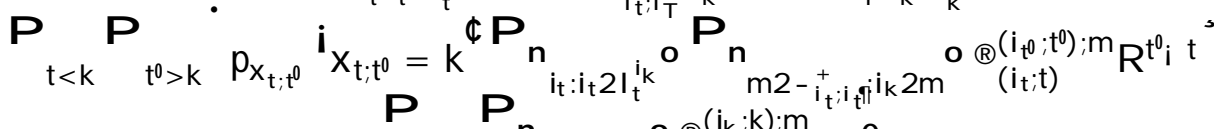

$$
\begin{aligned}
& i_{t<k}^{P} n_{i_{t}: i_{t} 2 I_{t}^{i_{k}}} \circ \mathbb{R}_{\left(i_{t} ; t\right)}^{\left(i_{k} ; k\right) ; m}=0
\end{aligned}
$$

Note that if $k=T_{i}$ 1; the constraint for $\phi_{i_{T_{i} 1}}$ the constraint for $\phi_{\mathrm{i}_{k}}$ is

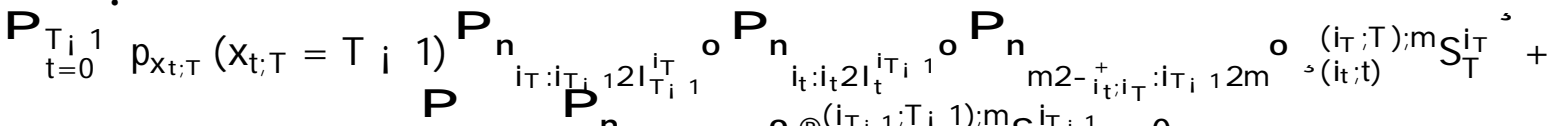

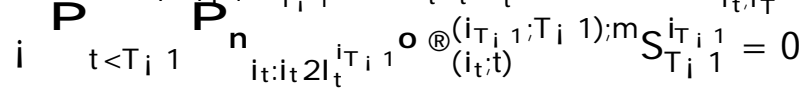

The constraint for $\mathrm{B}_{\mathrm{i}_{\mathrm{T}_{1} 1}}$ is

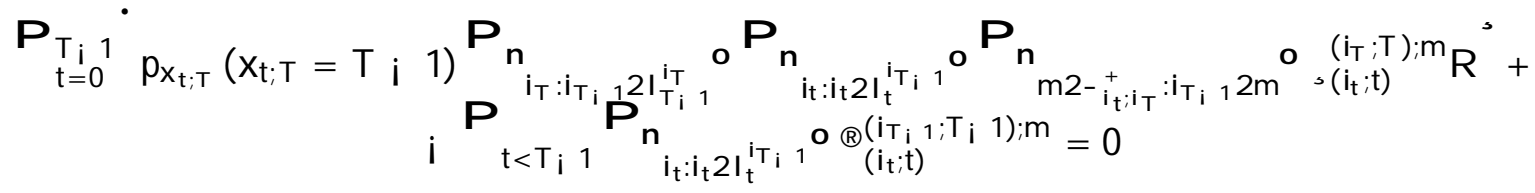

The left member of each constraint is a linear combination of the variables of the dual problem. The right member is equal to $S_{0}$ and 1 for the dual constraints associated with the variables $\phi 0$ and $\mathrm{B}_{0}$, respectively: For the remaining constraints the right member is equal to zero. First, let us consider only the constraints associated with primal variables $\$$ 's. For a given $\mathrm{i}_{\mathrm{T}}$ the terms involving, in the dual constraints regarding $\phi_{\mathrm{i}_{\mathrm{k}}}$ is

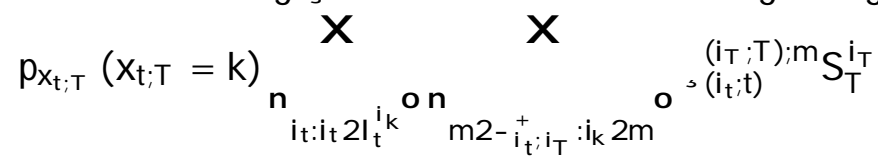


Summing up all the constraints that concern $\phi_{i_{k}}$ with $i_{k} 2 I_{k}$ the term associated with and the term associated with $S_{T}^{i}$ is

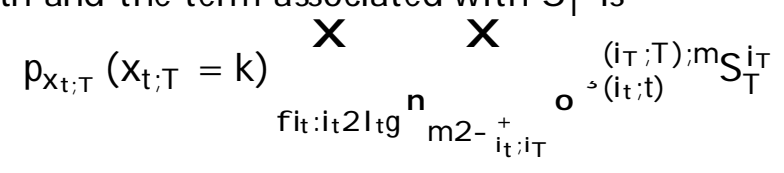

As,

$$
{ }_{k=t}^{X_{i}{ }^{1}} p_{x t ; T}\left(x_{t ; T}=k\right)=1 \text {; }
$$

summing for all $k, t$, the term associated with $S_{T}^{i_{T}}$ that is multiplying by $p_{x_{t} ; \mathrm{T}}\left(x_{t ; T}=:\right)$ is

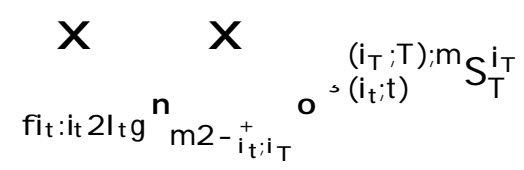

Hence, summing up over all constraints associated with primal variables $\varnothing$ ' $s$ we have that the terms in, associated with $S_{T}^{i_{T}}$ are

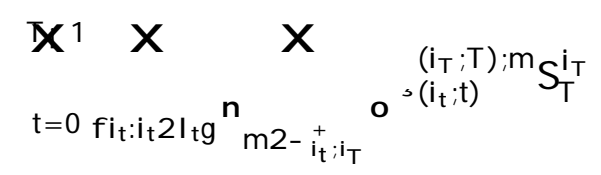

Therefore, the sum over all $S_{T}^{i_{T}}$ is

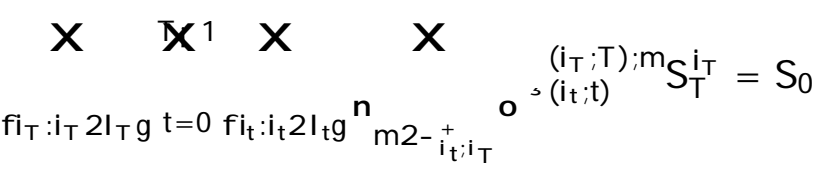

Still considering only the constraints associated with primal variables $\phi$ ' $S$; in what follows we describe the terms in ${ }^{\circledR}$. For a given $S_{t}^{i_{t}}$; the terms in $B$ are

$$
\begin{aligned}
& \left.\left.\right|_{\text {from the constraint } \phi_{i_{t}}} ^{X}\right\}
\end{aligned}
$$

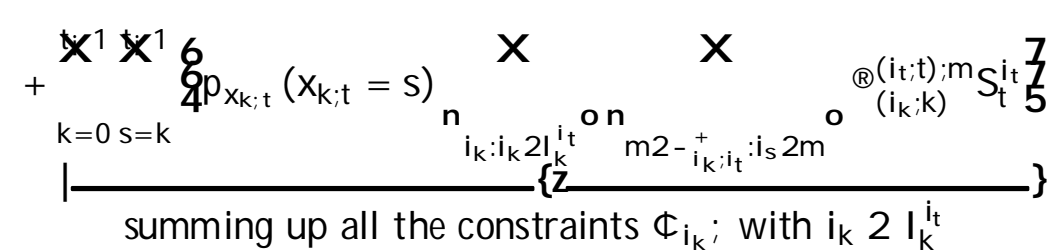


As,

$$
x_{s=k}^{1} p_{x_{k} ; t}\left(x_{k ; t}=s\right)=1
$$

the above equations sum up to zero. Summing up over all $S_{t}^{i t}$ a zero will also be obtained. Hence, if all dual constraints that concern $S_{t}^{i_{t}}$ are summed up, the following relation is obtained:

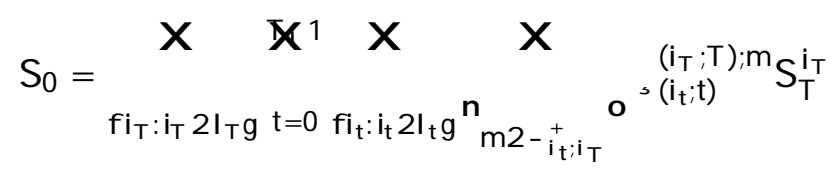

Now, proceeding in a similar way but considering the dual constraints associated with $B \$$ : Because the right member of the constraints is equal to 0 , excepting the one associated $\mathrm{B}_{0}$; we multiply each constraint by a constant. The constraint associated with the variable $B_{i_{k}}$ is multiplied by $R^{k}$ : Then, all the constraints associated with $\mathrm{B} \$$ are summed up, and

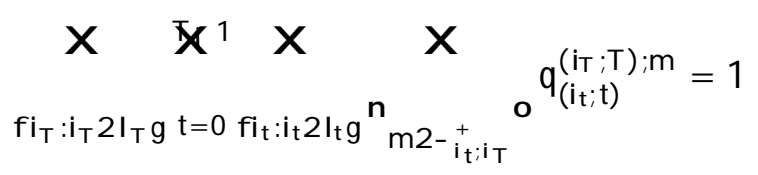

where

$$
q_{\left(i_{t} ; t\right)}^{\left(i_{T} ; T\right) ; m}=R^{T_{i} t},\left(i_{T} ; T\right) ; m:
$$

Denoting,

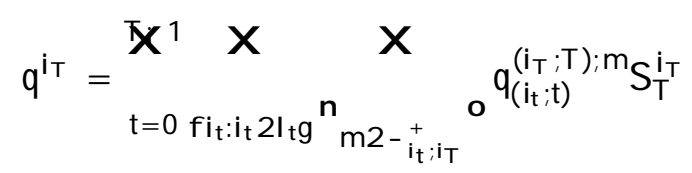

equation (4) can be written as

$$
S_{0}=\frac{1}{R^{\top}} \underset{f i_{T}: i_{T} 2 I_{T} g}{X} q^{j \top} S_{T}^{i_{T}}
$$

with

$$
\underset{\text { fіт :іт 2І т g }}{X} q^{\dot{\top} \tau=1}
$$




\section{A.2 Proof of theorem 10 with $T=3$}

Proof. As the problem sketched in the example of section to obtain the upper bound is a linear programming problem, considering $S_{t}^{i t}=U^{t_{i}\left(i_{t i}{ }^{1}\right)} D^{i_{t} i}{ }^{1}$; its dual is written as follows:

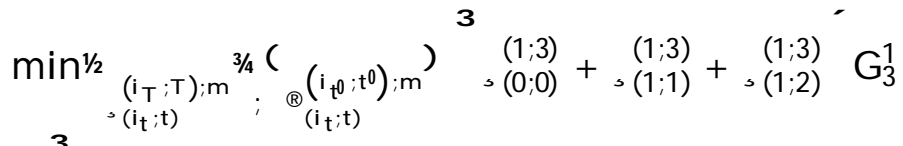

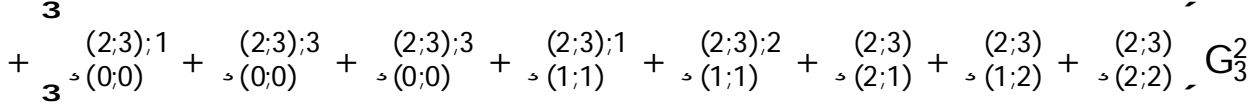

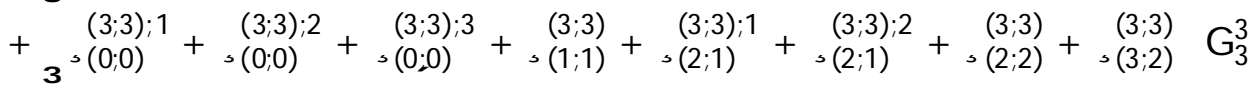

$$
\begin{aligned}
& +\quad(4 ; 3)+\stackrel{(4 ; 3 ; 3)}{(2 ; 0)}+\stackrel{(4 ; 3)}{(4 ; 2)} \mathrm{G}_{3}^{4}
\end{aligned}
$$

subject to the non-negativity constraints of the dual variables

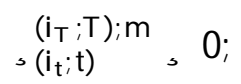

for all $i_{\mathrm{t}} 2 I_{\mathrm{t}}^{i_{\top}} ; i_{\top} 2 I_{T}$ and $t=0 ; 1$ and 2 ;

$$
\mathbb{R}\left(\mathrm{i}_{\left.\mathrm{t}_{t} ; \mathrm{t}\right)}^{\left.\mathrm{i}_{\mathrm{t}} 0 \mathrm{t}\right) ; \mathrm{m}}, 0\right. \text {; }
$$

for all $i_{t} 2 I_{t^{t}}^{i^{0}} ; i_{t^{0}} 2 I_{t^{0}}$ and $t^{0}=0 ; 1$ and 2 ; and subject to twelve equality constraints, each one associated with a variable of the primal problem. The constraint associated with $\phi 0$ is given by

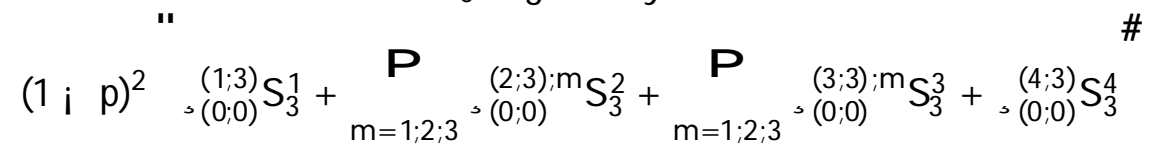

$$
\begin{aligned}
& +\mathbb{R}_{(0 ; 0)}^{(1 ; 1)} S_{1}^{1}+\mathbb{R}_{(0 ; 0)}^{(2 ; 1)} S_{1}^{2}+ \\
& +\left(1 \text { i p) }{ }^{h} \mathbb{R}_{(0 ; 0)}^{(1 ; 2)} S_{2}^{1}+{ }^{3} \mathbb{R}_{(0 ; 0)}^{(2 ; 2) ; 1}+\mathbb{R}_{(0 ; 0)}^{(2 ; 2) ; 2} S_{3}^{2}+\mathbb{R}_{(0 ; 0)}^{(3 ; 2)} S_{2}^{3^{i}}=S_{0}\right.
\end{aligned}
$$

The constraint associated with $B_{0}$ is given by

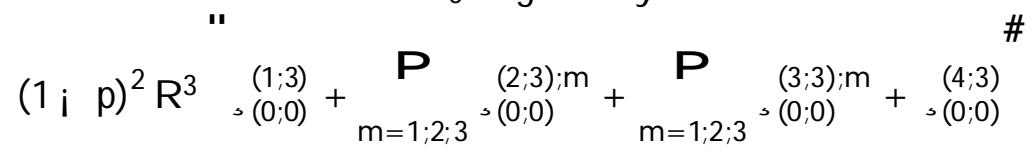

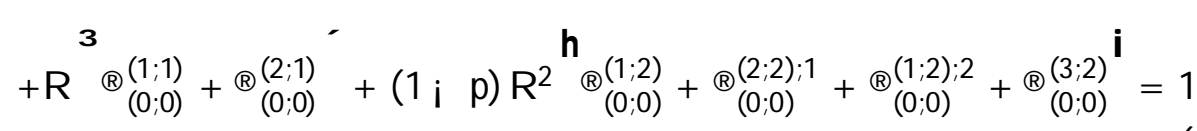


The constraint associated with $\pitchfork \frac{1}{1}$ is given by

$$
\begin{aligned}
& \left(1 \text { i p) }{ }_{,(1 ; 1)}^{(1 ; 3)} S_{3}^{1}+{ }_{m=1 ; 2}^{P}{ }_{(2 ; 1)}^{(2 ; 3) ; m_{3}} S_{3}^{2}+{ }_{,(1 ; 1)}^{(3 ; 3)} S_{3}^{3}+\right.
\end{aligned}
$$

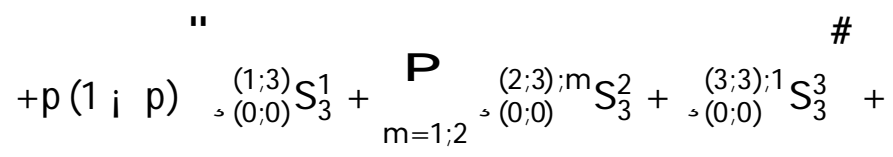

$$
\begin{aligned}
& i \mathbb{A}_{(0 ; 0)}^{(1 ; 1)} S_{1}^{1}+\mathrm{p}^{\mathrm{h}}\left(\mathbb{Q}_{0 ; 0)}^{(1 ; 2)} S_{2}^{1}+\mathbb{A}_{(0 ; 0)}^{(2 ; 2) ; 1} S_{2}^{i}+\mathbb{Q}_{(1 ; 1)}^{(1 ; 2)} S_{2}^{1}+\mathbb{Q}_{(1 ; 1)}^{(2 ; 2)} S_{2}^{2}=0\right.
\end{aligned}
$$

The constraint associated with $B_{1}^{1}$ is given by

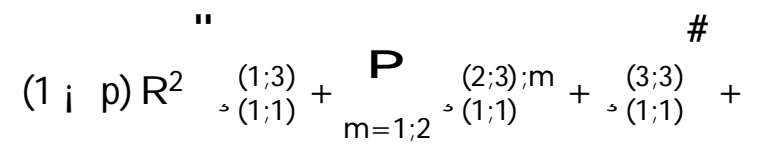

$$
\begin{aligned}
& +p\left(1 ; \text { p) } R^{2} \stackrel{(1 ; 3)}{\circ(0 ; 0)}+\underset{m=1 ; 2}{P} \underset{(2 ; 3) ; m}{(0 ; 0)} \stackrel{\substack{(3 ; 3) ; 1 \\
(0 ; 0)}}{\#}+\right.
\end{aligned}
$$

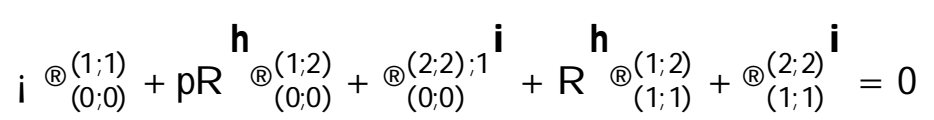

The constraint associated with $\phi_{1}^{2}$ is given by

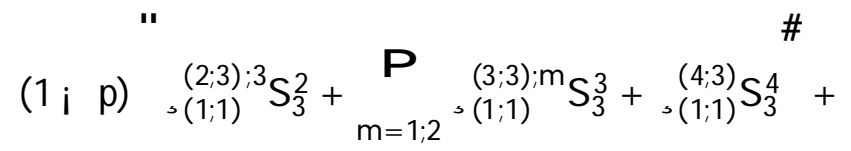

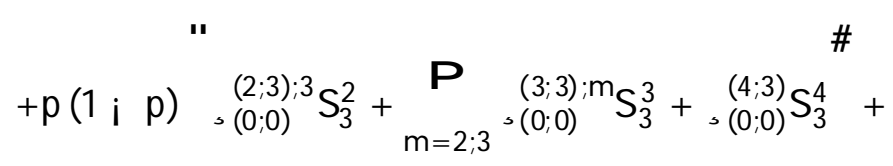

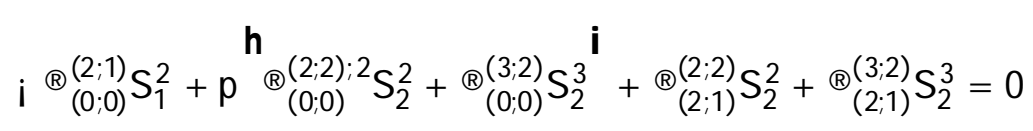


The constraint associated with $B_{1}^{2}$ is given by

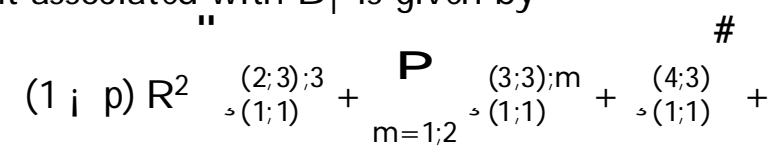

$$
\begin{aligned}
& +p\left(1 ; \quad \text { p) } R^{2} \stackrel{(2 ; 3) ; 3}{,(0 ; 0)}+\underset{m=2 ; 3}{P} \underset{(0 ; 0)}{(3 ; 3) ; m}+\underset{,(0 ; 0)}{\#}+\right.
\end{aligned}
$$

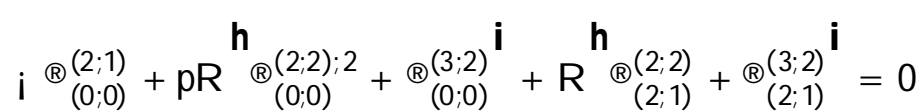

The constraint associated with $₫ \frac{1}{2}$ is given by

$$
\begin{aligned}
& \underset{,(1 ; 2)}{(1 ; 3)} U^{3} S_{0}+,{ }_{,(1 ; 2)}^{(2 ; 3)} U^{2} D S_{0}+p, \underset{(1 ; 3)}{(1 ; 1)} U^{3} S_{0}+, \underset{(1 ; 1)}{(2 ; 3) ; 1} U^{2} D S_{0} \\
& 3
\end{aligned}
$$

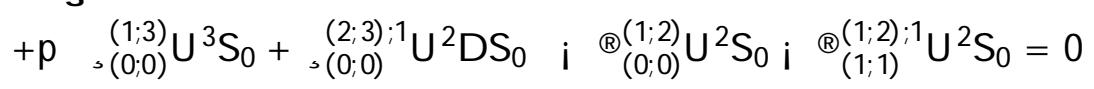

The constraint associated with $B_{2}^{1}$ is given by

$$
\begin{aligned}
& \mathrm{R}_{(1 ; 3)}^{\mathrm{h}_{(1 ; 2)}+{ }_{(2 ; 3)}+\mathrm{i}_{(1 ; 2)}}+\mathrm{h}_{(1 ; 3)}+{ }_{(2 ; 1)}^{(2 ; 3) ; 1} \mathrm{i}_{(1 ; 1)}
\end{aligned}
$$

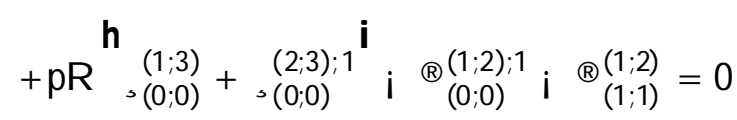

The constraint associated with $\phi \frac{2}{2}$ is given by

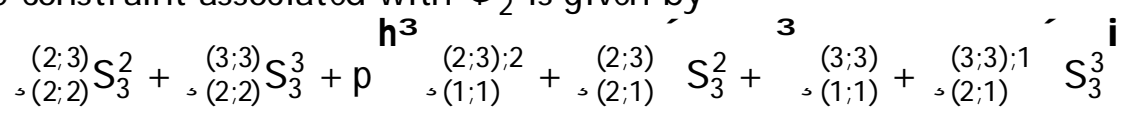

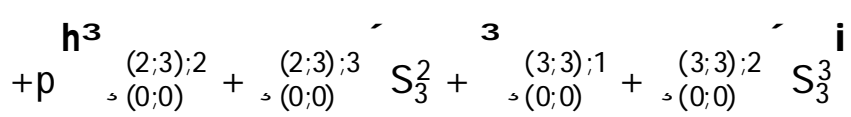

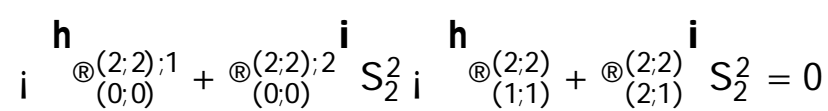

The constraint associated with $B_{2}^{2}$ is given by

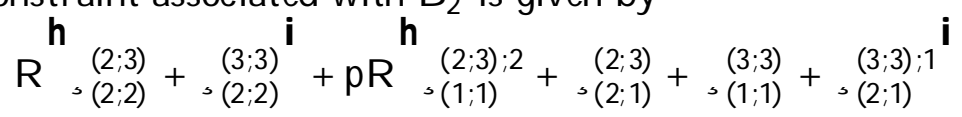

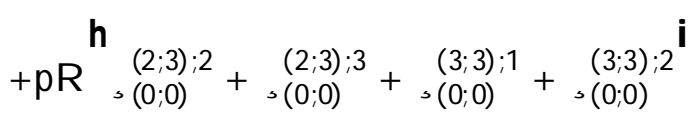

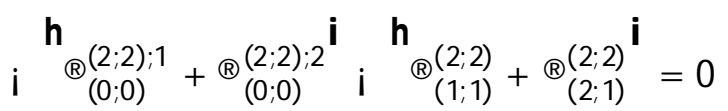


The constraint associated with $\pitchfork \frac{3}{2}$ is given by

$$
\begin{aligned}
& \underset{.(3 ; 2)}{(3 ; 3)} U D^{2} S_{0}+{ }_{,(3 ; 2)}^{(4 ; 3)} D^{3} S_{0}+p_{,(2 ; 1)}^{h}(3 ; 3) ; 2 U D^{2} S_{0}+,{ }_{(2 ; 3)}^{(4 ; 3)} D^{3} S_{0}
\end{aligned}
$$

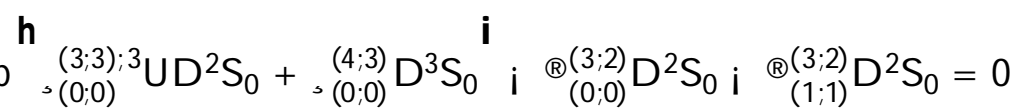

The constraint associated with $B_{2}^{3}$ is given by

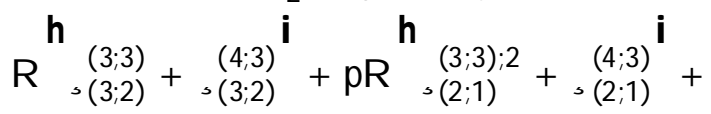

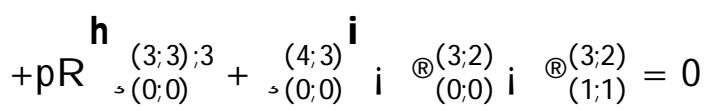

Summing up equations (5), (7), (9), (11),(13) and (15) we obtain

$$
\begin{aligned}
& \mathrm{S}_{0}=^{3} \underset{(1 ; 3)}{(1 ; 0)}+, \underset{(1 ; 1)}{(1 ; 3)}+\stackrel{(1 ; 3)}{(1 ; 2)} \mathrm{S}_{3}^{1}+
\end{aligned}
$$

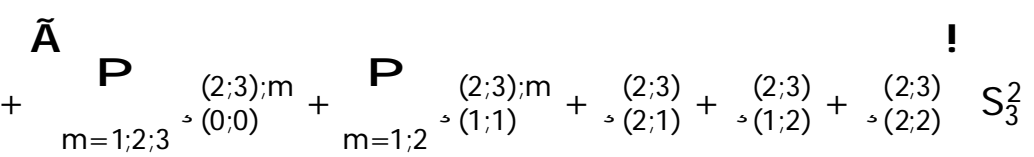

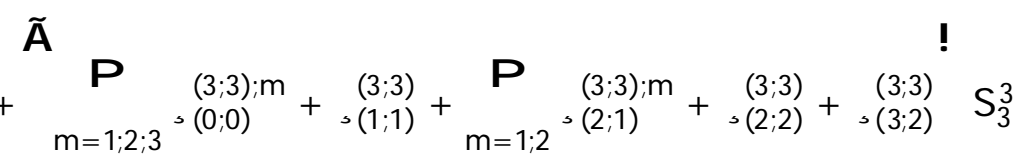

$$
\begin{aligned}
& \begin{array}{r}
3 \\
+\quad(4 ; 3) \\
.(0 ; 0)
\end{array}+(2 ; 3)+,(4 ;)+(3 ; 2) \quad S_{3}^{4}
\end{aligned}
$$

Multiplying equations (8) and (10) by $R$ and equations (12), (14) and (15) by $R^{2}$ and then summing up with equation (6) we obtain

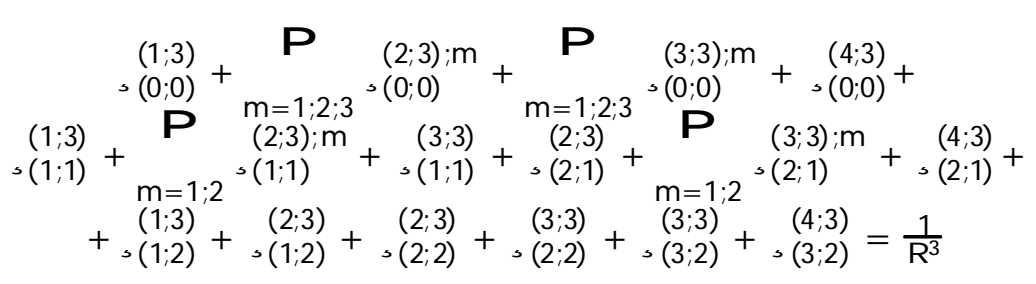

Hence, denoting

$$
q_{\left(i_{t} ; t\right)}^{\left(i_{t} ; t^{0}\right)}=R^{3}, \underset{\left(i_{t} ; t\right)}{\left(i_{t} 0 ; t 9\right.}
$$


and

$$
\begin{aligned}
& \begin{array}{c}
q^{1}=q_{\left(p_{0}\right)}^{(1 ; 3)}+q_{(; 1)}^{(1 ; 3)}+q_{(1 ; 2)}^{(1 ; 3)} \\
q^{2}={ }_{m=p^{2 ; 2 ; 3}} q_{(0 ; 0)}^{(2 ; 3) ; m}+{ }_{m=1 ; 2}^{(2 ; 3) ; m}+q_{(2 ; 1)}^{(2 ; 3)}+q_{(1 ; 2)}^{(2 ; 3)}+q_{(2 ; 2)}^{(2 ; 3)} \\
q^{3}={ }_{m=1 ; 2 ; 3} q_{(0 ; 0)}^{(3 ; 3) ; m}+q_{(1 ; 1)}^{(3 ; 3)}+{ }_{m=1 ; 2} q_{(2 ; 1)}^{(3 ; 3) ; m}+q_{(2 ; 2)}^{(3 ; 3)}+q_{(3 ; 2)}^{(3 ; 3)}
\end{array} \\
& q^{4}=q_{(0 ; 0)}^{(4 ; 3)}+q_{(2 ; 1)}^{(4 ; 3)}+q_{(3 ; 2)}^{(4 ; 3)}
\end{aligned}
$$

we can rewrite (17) as

$$
S_{0}=\frac{1}{R^{3}}{ }^{f}{ }^{1} S_{3}^{1}+q^{2} S_{3}^{2}+q^{3} S_{3}^{3}+q^{4} S_{3}^{4^{\alpha}}
$$

with $q$ are de ed above and $q^{1}+q^{2}+q^{3}+q^{4}=1$ :

Taking into consideration equation (10), equation (9) can be rewritten as

$$
S_{2}^{1}=\frac{1}{R}{ }^{h /(1 ; 3)} S_{\frac{1}{(1 ; 2)}}^{1}+\frac{1 /(2 ; 3)}{(1 ; 2)} S_{3}^{2}
$$

where

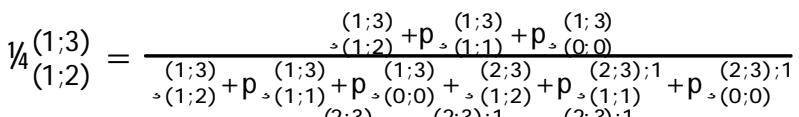

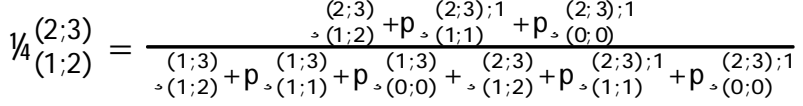

and $1 /(1 ; 3)+1 / 2)+1(2 ; 3)=1$ : Proceeding in an analogous way with constraints (13), (14), (15) and (16) we obtain

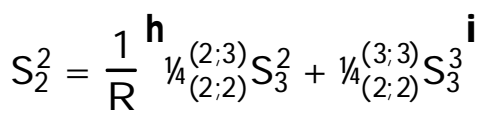

where

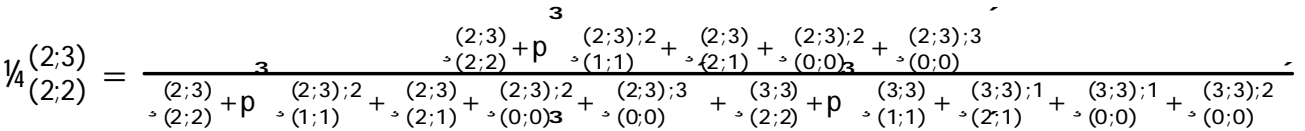

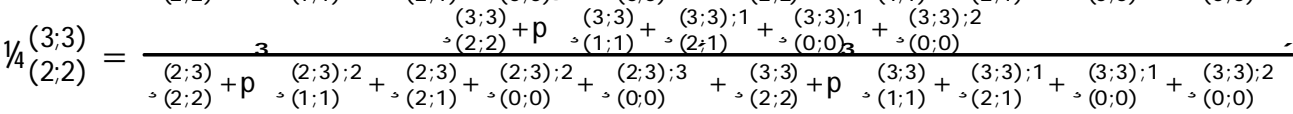

with $1 /(2 ; 3)+1 /(3 ; 2 ; 2)=1$; and

$$
\mathrm{S}_{2}^{3}=\frac{1}{\mathrm{R}}{ }^{\mathrm{h}}{ }^{1 /(3 ; 3)}(3) \mathrm{S}_{3}^{3}+1 /\left(\frac{1}{(3 ; 2)} \mathrm{S}_{3}^{4}\right.
$$


where

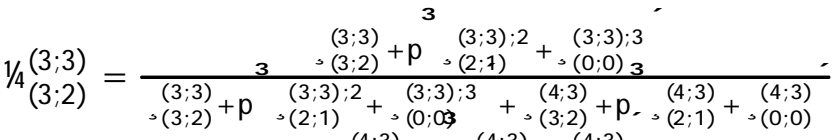

$$
\begin{aligned}
& 1 / 4 ;(4 ; 3)=\frac{{ }_{(3 ; 2)}}{.(3 ; 2)+p_{,(2 ; 1)}^{(4 ; 3)}+\left(\begin{array}{l}
(4 ; 3) \\
(0 ; 0)
\end{array}\right.}
\end{aligned}
$$

with $1 /(3 ; 3)+1 / 4(4 ; 3)=1$ :

M or eover, using equations (7), (8), (9) and (10) we have

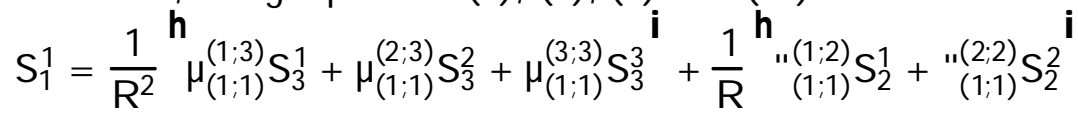

where

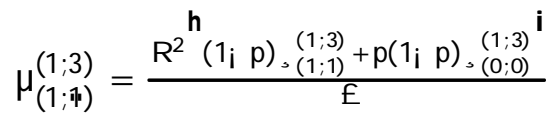

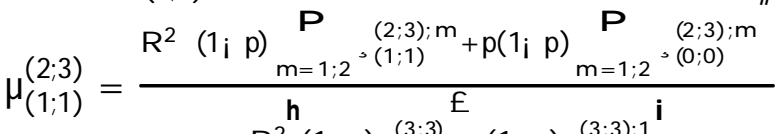

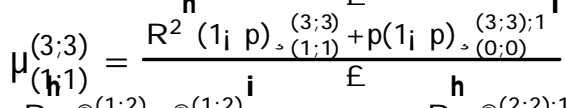

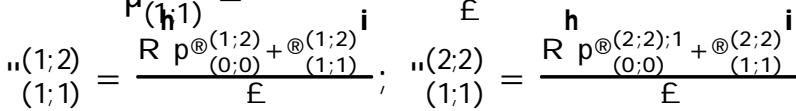

$$
\begin{aligned}
& f=R^{2} \underset{2}{h}(1 ; p), \underset{(1 ; 1)}{(1 ; 3)}+p(1 ; p), \underset{(0 ; 0)}{(1 ; 3)}
\end{aligned}
$$

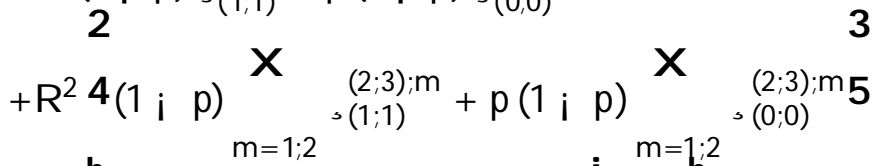

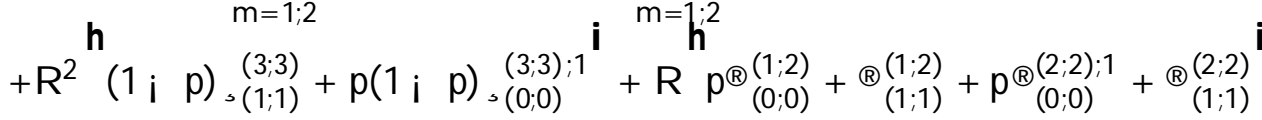

and

$$
\begin{aligned}
& S_{1}^{2}=\frac{1}{R^{2}}{ }^{h} \mu_{(2 ; 1)}^{(1 ; 3)} S_{3}^{2}+\mu_{(2 ; 1)}^{(2 ; 3)} S_{3}^{3}+\mu_{(2 ; 1)}^{(3 ; 3)} S_{3}^{4}+\frac{1}{R}{ }^{{ }^{\prime \prime}(1 ; 2)} S_{(2 ; 1)} S_{2}^{2}+{ }^{~}{ }_{(2 ; 1)}(2 ; 2) S_{2}^{3} \text {, with }
\end{aligned}
$$

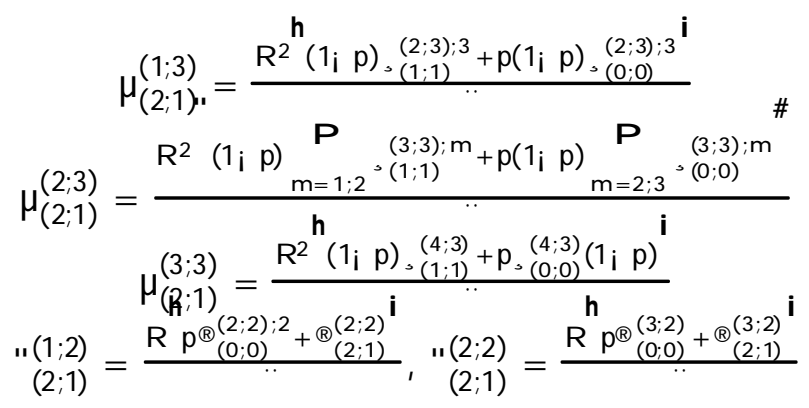




$$
\begin{aligned}
& .=R^{2}{ }_{2}^{h}(1 ; p), \underset{(1 ; 1)}{(2 ; 3) ; 3}+p(1 ; p) \underset{\sim(0 ; 0)}{(2 ; 3) ; 3^{i}}
\end{aligned}
$$

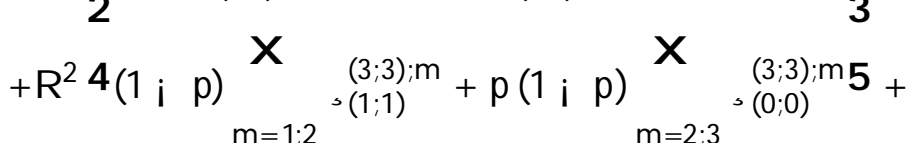

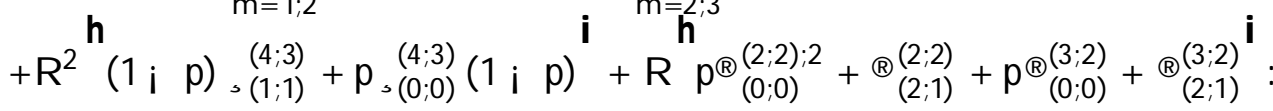

\section{A.3 Upper Bound for $\mathrm{T}=2$}

For $\mathrm{T}=2$ the problem that must be solved to ${ }^{-}$nd the upper bound of the arbitrage free range of variation is the following

$$
\mathrm{C}_{\mathrm{u}}=\min _{\mathrm{f} \Phi_{0} ; \mathrm{B}_{0} ; \uparrow_{1}^{1} ; \mathrm{B}_{1}^{1} ; \varangle_{1}^{2} ; \mathrm{B}_{1}^{2} \mathrm{~g}} \uparrow 0 \mathrm{~S}_{0}+\mathrm{B}_{0}
$$

subject to the conditions of positive expected payo ${ }^{\circledR}$ at time $t=0$;

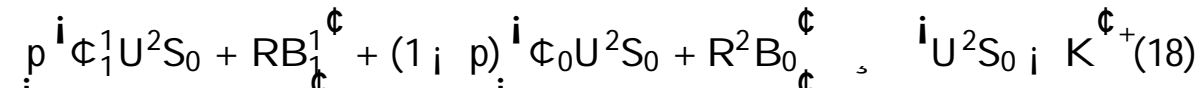

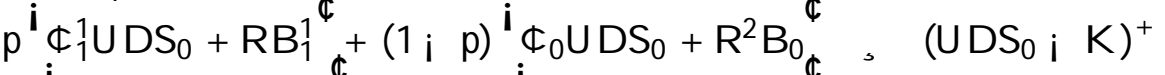

$$
\begin{aligned}
& p^{i} \phi_{1}^{2} U D S_{0}+R B_{1}^{2}+(1 ; p){ }^{i} \phi D^{2} S_{0}+R^{2} B_{0}{ }^{\phi}, \quad\left(U D S_{0} i K\right)^{+} \\
& p^{i} \phi_{1}^{2} D^{2} S_{0}+R B_{1}^{2}+(1 ; p){ }^{i} \phi{ }_{0} D^{2} S_{0}+R^{2} B_{0}{ }^{\phi}, \quad{ }^{i} D^{2} S_{0} i K^{\phi_{+}}(19)
\end{aligned}
$$

positive expected payo ${ }^{\circledR}$ at time $\mathrm{t}=1$;

$$
\begin{aligned}
& { }^{i} \phi \frac{1}{1} U^{2} S_{0}+R B_{1}^{1}{ }_{1}^{\phi}, \quad{ }^{i} U^{2} S_{0} i K^{\phi_{+}} \\
& \mathrm{i}_{\phi}{ }_{1}^{1} \mathrm{UDS}_{0}+\mathrm{RB}_{1}^{1}{ }_{1} \Phi, \quad\left(\mathrm{UDS}_{0} \mathrm{i} K\right)^{+} \\
& \mathrm{i}_{\phi}{ }_{1} \mathrm{UDS}_{0}+\mathrm{RB}_{1}^{2} \phi, \quad\left(U D S_{0} \mathrm{i} K\right)^{+} \\
& \mathrm{i}{ }_{1}^{2} \mathrm{D}^{2} \mathrm{~S}_{0}+R \mathrm{~B}_{1}^{2}{ }^{\Phi},{ }^{\mathrm{i}} \mathrm{D}^{2} \mathrm{~S}_{0} \mathrm{i} \mathrm{K}^{\phi_{+}}
\end{aligned}
$$

and self-" nancing,

$$
\begin{aligned}
& \phi_{1}^{1} U S_{0}+B_{1}^{1} \cdot \phi_{0} U_{0} S_{0}+R_{0} \\
& \phi_{1}^{2} D S_{0}+B_{1}^{2} \cdot \phi_{0} D S_{0}+R B_{0}:
\end{aligned}
$$


Construct the Lagrangean

$$
\begin{aligned}
& L=\$ S_{0}+h_{i} B_{0}+
\end{aligned}
$$

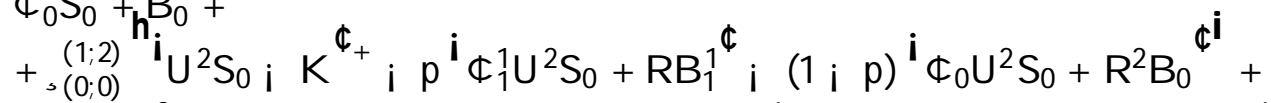

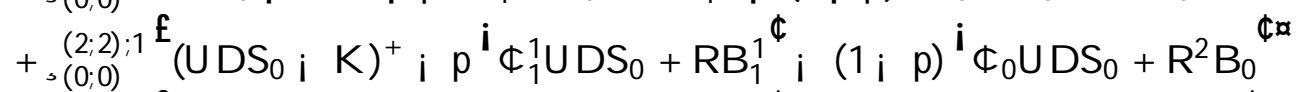

$$
\begin{aligned}
& +,(2 ; 2) ; 2{ }^{f}\left(U D S_{0} i K\right)^{+} i p^{i} \phi \frac{2}{1} U D S_{0}+R_{1}^{2}{ }^{\phi} i(1 ; p){ }^{i} \phi D^{2} S_{0}+R^{2} B_{0}{ }^{\phi \alpha}
\end{aligned}
$$

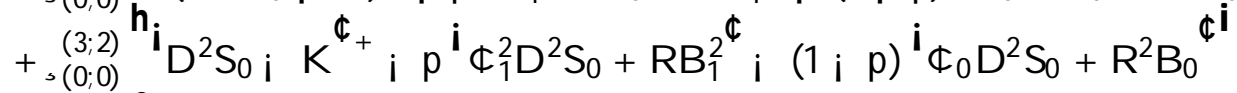

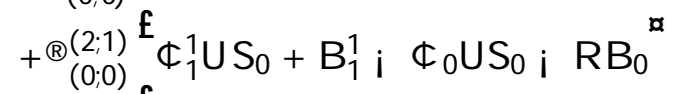

$$
\begin{aligned}
& +\mathbb{R}_{0 ; 0)}^{(1 ; 1)} f_{\phi}{ }_{1}^{2} \mathrm{D} S_{0}+\mathrm{B}_{1}^{2} \mathrm{i}\left(\phi \mathrm{DDS}_{0}+\mathrm{RB}_{0}\right)^{\not}
\end{aligned}
$$

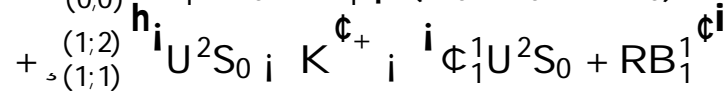

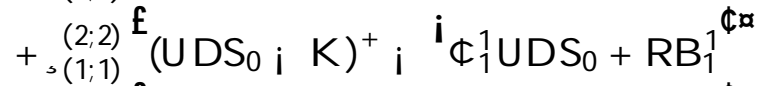

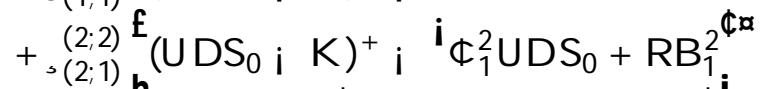

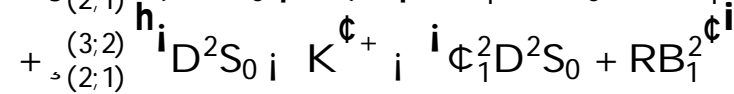

The solution is characterized by

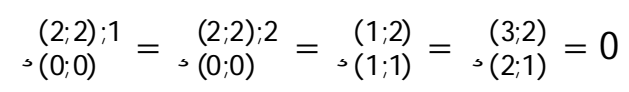

and

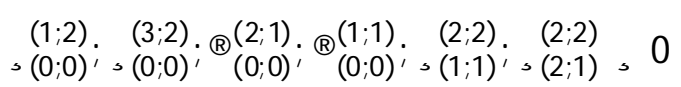

Using the fact that equations (18), (19), (22), (23), (20) and (21) are binding the optimal values $\phi_{0}^{\alpha} ; B_{0}^{\alpha} ; ; \phi_{1}^{1 \alpha} ; B_{1}^{1 \alpha} ; \phi_{1}^{2 \alpha} ; B_{1}^{2 \alpha}$ can be obtained. In particular, $\phi_{0}^{\infty}$ e $B_{0}^{\alpha}$ are given by 


$$
\begin{aligned}
& \phi_{0}^{\infty}=\frac{{ }^{i} U^{2} S_{0} i K^{\phi_{+}}{ }^{h}\left(U_{i} R\right)\left(R_{i} D\right)+p\left(R_{i} D\right)^{2^{i}}}{i^{i} D^{2} S_{0 i} K^{\phi}} \\
& \frac{i^{i} D^{2} S_{0} i K^{\phi_{+}}{ }^{h}(U ; R)(R ; D)+p(R ; U)^{2}}{S^{i} \alpha} \\
& \frac{i \mathrm{p}\left(U^{2} S_{0} i K\right)^{+}{ }_{i}^{f} 2 \mathrm{DR}+2 R U+D^{2} i U^{2^{\alpha}}}{S_{0} \alpha}
\end{aligned}
$$

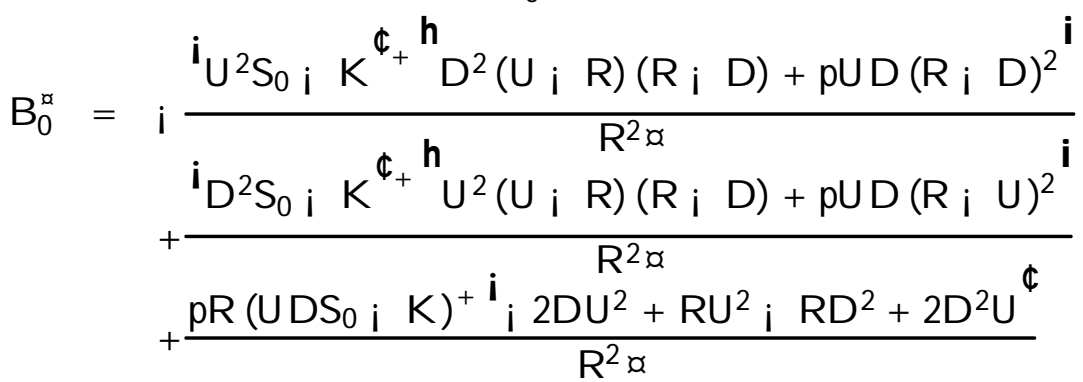

where

$$
\begin{aligned}
\alpha= & U^{2}{ }^{f}\left(U{ }_{i} R\right)(R ; \quad D) i p^{i}{ }_{i} R^{2}+4 R D i D U^{\phi \alpha} \\
& i D^{2} \stackrel{f}{(U}\left(U_{i} R\right)(R i \quad D) i p^{i}{ }_{i} R^{2}{ }_{i} U D+4 R U^{\phi \alpha}
\end{aligned}
$$

The remaining equations are also satis ${ }^{-}$ed.

As a result, after some trivial algebra, we obtain

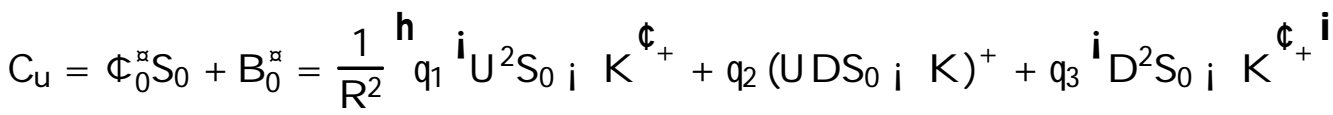

with

$$
\begin{aligned}
& q_{2}=\frac{(U ; R)(R ; D){ }^{i} R^{2} i D^{2}+p(R ; D)^{2}{ }^{i} R^{2} i U D^{\phi}}{\varnothing}
\end{aligned}
$$

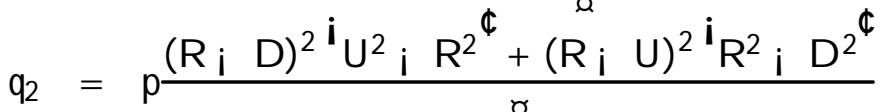

$$
\begin{aligned}
& \alpha_{B}=\frac{(U ; R)(R ; D){ }^{i} U^{2} i R^{2^{\Phi}}+p(R ; U)^{2 i} U D_{i} R^{2^{\Phi}}}{n}
\end{aligned}
$$

It is easy to check that $q_{1} ; q_{2} ; q_{\beta}, 0$ and $q_{1}+q+q_{\beta}=1$ : 


\section{A.4 Proofs of the Properties and Examples}

\section{A.4.1 Property 3}

1. P roof. Let the set of admissible solutions that characterize the upper bound for the case p $2(0 ; 1)$ be denoted by $A(p)$;where $A(p)$ is a corresp ondence such that

$$
A(p):[0 ; 1] ! R^{t(t+1)}:
$$

The portfolio $(\phi ; B)=\left(\phi i_{t} ; B_{i_{t}}\right)_{i_{t} 2 l_{t} ; t=0 ; \ldots ; T_{i} 1} 2 R^{t(t+1)}$ is said to be an admissible solution for the problem de ned in section 4.1.1 if $(\phi ; B) 2$ A (p) :

Moreover, let $A(p=0)$ and $A(p=1)$ denote, respectively, the admissible solutions for the problems characterizing the upper bound in the case $p=0$ and $p=1$; presented in section 2.1.

By the Theorem of the M aximum, ${ }^{13}$ if the constraint correspondence $A(p)$ is continuous and if the objective function is continuous on $p$, then the value of the objective function in the optimum is al so continuous on $\mathrm{p}$.

First consider the case $p$ ! 1 : In this case, $\lim _{p !} A(p)=A(p=1)$ : Hence,

$$
\lim _{\mathrm{p} !} C_{\mathrm{u}}^{\mathrm{p}}=\mathrm{C}_{\mathrm{u}}^{\mathrm{p}}(\mathrm{p}=1)=\mathrm{C}_{\mathrm{u}}^{1}
$$

However, the same does not apply when $p$ ! 0 : In this case

$$
\lim _{p !} A(p) \mu A(p=0)
$$

which implies that

$$
C_{u}^{p}(p=0), C_{u}^{0}:
$$

However, using property 1 , we conclude $C_{u}^{p}(p=0)=C_{u}^{0}$ :

Example 21 (for $T=2$.) When $p$ ! 0 , and in the case of a Call Option, which means that the market is completely illiquidity, the optimal values of $\$ 0$ and $\mathrm{B}_{0}$ in expression (24) converge to

$$
\begin{aligned}
& \phi_{0}^{\infty}=\frac{{ }^{i} U^{2} S_{0} i K^{\Phi_{+}} i^{i} D^{2} S_{0} i K^{\Phi_{+}}}{S_{0}\left[U^{2}{ }^{i} D^{2}\right]} \\
& B_{0}^{\infty}=\frac{U^{2}{ }^{i} D^{2} S_{0} i K^{\Phi_{+}} i D^{2} U^{2} S_{0} i K^{\Phi_{+}}}{R^{2}\left(U^{2} i D^{2}\right)}
\end{aligned}
$$

\footnotetext{
${ }^{13}$ See, for instance, Mas-Collel et al. (1995), page 963.
} 
then,

$$
\begin{aligned}
\lim _{p ! 0} C_{u}^{p} & ={ }_{0}^{x_{0}} S_{0}+B_{0}^{\alpha} \\
& =\frac{1}{R^{2}}{\frac{R^{2} i D^{2}}{U^{2} i D^{2}}}^{\cdot i^{2} S^{2} S_{0} i K^{\phi_{+}}+{ }^{\mu} \frac{U^{2} i R^{2}}{U^{2} i D^{2}}{ }^{i} D^{2} S_{0} i K}{ }^{\Phi_{+}} ;
\end{aligned}
$$

i.e., $\lim _{p !}{ }_{0} C_{u}^{p}=C_{u}^{0}$ : On the other hand, when $p$ ! 1 , which means that there is no illiquidity, the optimal values of $\phi_{0}$ and $B_{0}$ in expression (24) converge to

$$
\begin{aligned}
& \phi_{0}^{x}=\frac{{ }^{i} U^{2} S_{0} i K^{\phi_{+}}(R ; D) i^{i} D^{2} S_{0} i K^{\phi_{+}}\left(U_{i} R\right)+\left(U D S_{0} i K\right)^{+}\left(U_{i} 2 R+D\right)}{S_{0} R(U ; D)^{2}} \\
& B_{0}^{\infty}=i \frac{D(R ; D)}{R^{2}(U ; D)^{2}}{ }^{i} U^{2} S_{0} i K^{\phi_{+}}+\frac{U(U ; R)}{R^{2}(U ; D)^{2}}{ }^{i} D^{2} S_{0} i K^{\phi_{+}} \\
& +\frac{[i D(U ; R)+U(R ; D)]}{R^{2}(U ; D)^{2}}\left(U^{2} S_{0} i K\right)^{+}
\end{aligned}
$$

then,

$$
\begin{aligned}
& \lim _{p !} C_{u}^{p}=\phi{ }_{0}^{x} S_{0}+B_{0}^{x}
\end{aligned}
$$

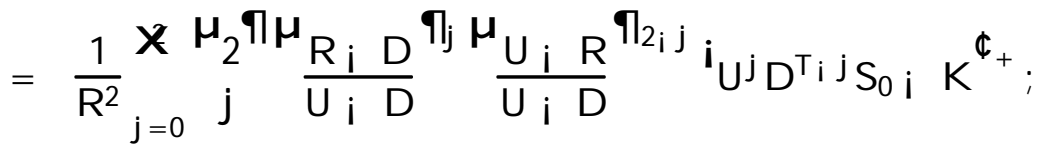

i.e., $\lim _{p !}{ }_{0} C_{u}^{p}=C_{u}^{1}$ :

\section{A.4.2 Property 4}

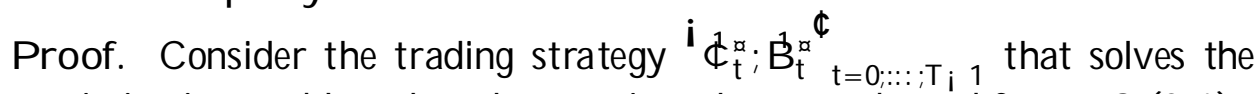
maximization problem that characterizes the upper bound for a p2 $(0 ; 1)$ :

Fix a given path $\mathrm{w}$, such that $\left(\mathrm{i}_{\mathrm{t}}\right)_{\mathrm{t}=0 ; \ldots ; ; \mathrm{T}} 2 \mathrm{w}$ : For each path we have $\mathrm{T}$ superreplicating constraints, one for each t 2 f0;:: ; $\mathrm{T}_{\mathrm{i}} \mathrm{lg}$ : For $\mathrm{T}_{\mathrm{i}} \mathrm{1}$;

$$
\stackrel{1}{C_{i_{T i} 1}^{\alpha}} S_{T}^{i_{T}}+R B_{i_{T_{i} 1}}^{\alpha}, G_{T}^{i_{T}}
$$

for $\mathrm{t}=\mathrm{T}_{\mathrm{i}_{3}} 2$

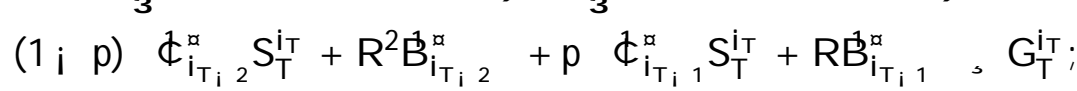


which can be rewritten as

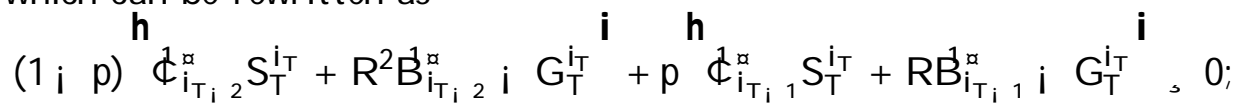

$$
\begin{aligned}
& \text { for } \mathrm{t}=\mathrm{T}_{\mathrm{i}}{ }_{3}^{3}
\end{aligned}
$$

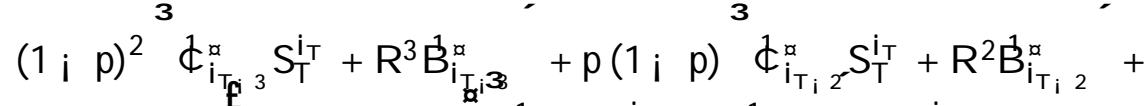

$$
\begin{aligned}
& +\stackrel{p}{f}(1 ; p)+p^{2^{\alpha}} \epsilon_{i_{T i 1}}^{\alpha} S_{T}^{i_{T}}+R B_{i_{T i 1}}^{\alpha}, G_{T}^{i_{T}} \text {; }
\end{aligned}
$$

which can be rewritten as

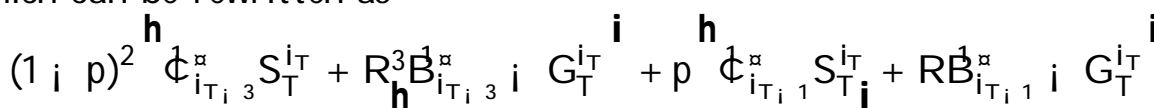

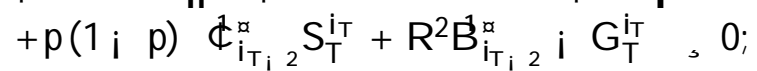

M ore generally, for $t$

$$
\begin{aligned}
& E_{i_{t}}=E_{i_{t+1}}+(1 i p)_{3}^{T_{i} t_{i} 1^{3}} \phi_{i_{t}}^{\alpha} S_{T}^{i_{T}}+R^{T i}{ }^{t^{2}} B_{i_{t}}^{\alpha}+
\end{aligned}
$$

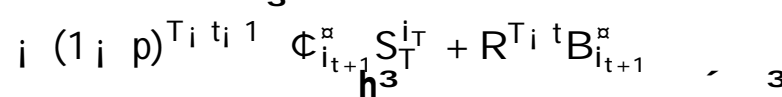

$$
\begin{aligned}
& =E_{i_{t+1}}+\left(\begin{array}{lll}
1 & p
\end{array}\right)^{T_{i} t_{i} 1} \phi_{i_{t}}^{\alpha} S_{T}^{i_{T}}+R^{T i}{ }^{t} B_{i_{t}}^{\alpha} i \quad \phi_{i_{t+1}}^{\alpha} S_{T}^{i_{T}}+R^{T_{i} t^{t}} B_{i_{t+1}}^{\alpha}{ }^{i}, 0
\end{aligned}
$$

B y backward induction since $T=t_{i} 1$; we can prove that $E_{i_{t+1}}$ is positive. Hence, if $E_{i_{t}}$ is positive for a given $p$ it will also be positive for another $p$; because when $p$ increases $(1 ; p)^{T} t_{i}{ }^{1}$ decreases. Hence, whatever the sign of

$$
{ }^{3} \phi_{i_{t}}^{\alpha} S_{T}^{i_{T}}+R^{T_{i}{ }^{t}} B_{i_{t}}^{\alpha}{ }^{\prime}{ }^{3} \phi_{i_{t+1}}^{\alpha} S_{T}^{i_{T}}+R^{T_{i}{ }^{t}} B_{i_{t+1}}^{\alpha}
$$

$\mathrm{E}_{\mathrm{i}}$ will remain positive.

The same applies for the self-` ${ }^{-}$nancing conditions.

\section{A.4.3 Property 5}

The upper bound of the ask price in a complete market can be written as

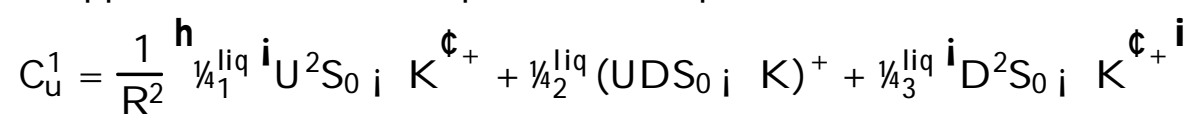


with

$$
\begin{aligned}
& { }_{1 / 4} \operatorname{liq}=\frac{\left(R_{i} D\right)^{2}}{\left(U_{i} D\right)^{2}} \\
& 1 / 4 q=\frac{\left(R_{i} D\right)\left(U_{i} R\right)}{\left(U_{i} D\right)^{2}} \\
& 1 /{ }_{4}^{i q}=\frac{\left(U_{i} R\right)^{2}}{\left(U_{i} D\right)^{2}}
\end{aligned}
$$

and the upper bound of the ask price in an illiquid market can be written as

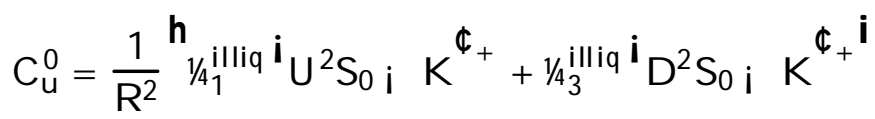

with

$$
\begin{aligned}
& \underset{4}{1 / \text { lliq }}=\frac{R^{2} i D^{2}}{U^{2} i D^{2}} \\
& \underset{3}{1 / l l i q}=\frac{U^{2} i R^{2}}{U^{2} i D^{2}}
\end{aligned}
$$

Hence, the upper bound for the ask price in a random dry market can be written

$$
C_{u}^{p}={ }_{B} C_{u}^{1}+(1 ; \quad \&) C_{u}^{0}
$$

with

$$
\begin{aligned}
\circledR & =p \frac{\frac{R\left(U_{i} D\right)^{2}}{\left(U_{i} R\right)(U+D)\left(R_{i} D\right)}}{1+p \frac{R\left(U_{i} D\right)^{2}}{\left(U_{i} R\right)(U+D)\left(R_{i} D\right)} i} i \\
1_{i} B & =\frac{h \frac{1 i p}{1+p \frac{R\left(U_{i} D\right)^{2}}{\left(U_{i} R\right)(U+D)\left(R_{i} D\right)} i 1}}{i}
\end{aligned}
$$

Taking the derivative of ${ }^{\circledR}$ with respect to $p$ we obtain $\frac{@ R}{@ D}=\frac{R(U ; D)^{2}(R+D)(U ; R)(U+D){ }^{i} R^{2} i D^{2}}{(U ; R)(U+D)\left(R^{2} i D^{2}\right)+p(R ; D)^{2} U(R+D)+(U ; R)^{2} D(R+D)} i \sigma_{2}, 0$ 
Hence, taking the derivative of $\mathrm{C}_{\mathrm{u}}^{0}$ with respect to $\mathrm{p}$ we conclude that $\mathrm{C}_{\mathrm{u}}^{0}$ is a decreasing function of $\mathrm{p}$;

$$
\begin{aligned}
& \frac{@ C_{u}^{p}}{\Phi}=\frac{@}{\Phi} C_{u}^{1} i \frac{@ R}{@ D} C_{u}^{0}
\end{aligned}
$$

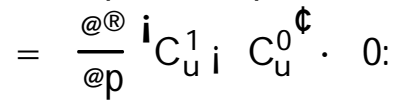

Taking the second derivative we can also conclude that $\mathrm{C}_{\mathrm{u}}^{\mathrm{p}}$ is a convex function with respect to $\mathrm{p}$;

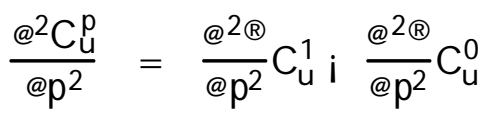

$$
\begin{aligned}
& =\frac{\mathfrak{a}^{2} \mathrm{i}}{\mathfrak{a}^{2}} \mathrm{C}_{\mathrm{u}}^{1} \mathrm{i} \mathrm{C}_{\mathrm{u}}^{0^{\phi}}
\end{aligned}
$$

as

$$
\frac{\mathfrak{Q}(\mathbb{a}}{\mathfrak{a}^{2}} \cdot 0
$$

we have

$$
\frac{\mathfrak{Q}^{2} C_{u}^{p}}{\mathfrak{p}^{2}}, 0:
$$

Alternative proof: After some algebra we obtain that following relation between ${ }^{\circledR}$ in equation $(25)$ and $\mathrm{p}$ :

$$
{ }^{\circledR}>\mathrm{p}
$$

So, as

$$
\mathrm{C}_{0}^{\text {ask liq }} \mathrm{i} \mathrm{C}_{0}^{\text {ask illiq } \cdot 0}
$$

we obtain,

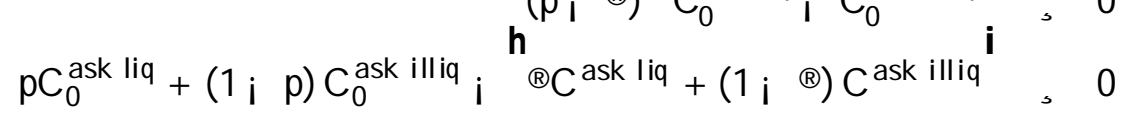

and follows that

$$
\mathrm{C}_{0}^{\text {ask }} \cdot \mathrm{pC}_{0}^{\text {ask liq }}+\left(1 \mathrm{i} \text { p) } \mathrm{C}_{0}^{\text {ask illiq. }}\right.
$$




\section{B Some Proofs on the Solution of the Lower B ound for Statistical Arbitrage Opportunities}

\section{B.1 Proof of theorem 12}

Proof. The problem that must be solved in order to ${ }^{-}$nd the upper bound of the range of variation of the arbitrage free value of an $E$ uropean derivative is a linear programming problem. Its dual problem is

$$
\max _{{ }_{.}{ }_{T}}{ }^{X+1},{ }_{j=1}^{i_{T}} G_{T}^{i_{T}}
$$

where, ${ }^{\mathrm{i}}{ }_{\mathrm{T}}$ is the sum of the dual variables associated with the positive expected payo ${ }^{\circledR}$ constraints that have the right member equal to $\mathrm{G}_{\mathrm{T}}^{\mathrm{i}}$, i.e.,

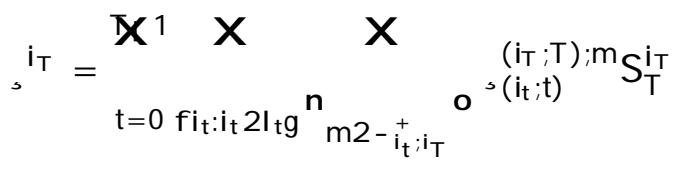

The - rst set of constraints is of nonnegativity of each dual variable, i.e,

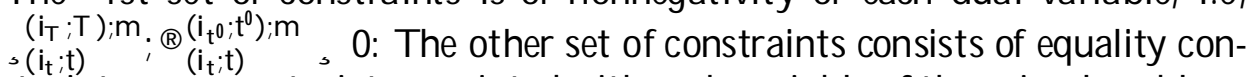
straints, one constraint associated with each variable of the primal problem. The other set are equality constraints which are equal to the ones obtained for the upper bound. Using the same argument as in the proof of theorem 1 the proof is straightforward.

\section{B.2 Lower Bound for $\mathrm{T}=2$}

For $\mathrm{T}=2$ the problem that must be solved to ${ }^{-}$nd the lower bound of the arbitrage free range of variation is the following

$$
C_{I}=\max _{f \notin 0 ; B_{0} ; \varangle_{1}^{1} ; B_{1}^{1} ; \uparrow_{1}^{2} ; B_{1}^{2} g} \$ 0 S_{0}+B_{0}
$$

subject to the conditions of positive expected payo ${ }^{\circledR}$ at time $t=0$;

$$
\begin{aligned}
& p^{i} \phi{ }_{1}^{1} U^{2} S_{0}+R B_{1}^{1}{ }_{1}^{\phi}+\left(\begin{array}{lll}
1 i & p
\end{array}{ }^{i} \phi U^{2} S_{0}+R^{2} B_{0}^{\phi} . \quad{ }^{i} U^{2} S_{0} i K^{\phi+}\right. \\
& p^{i} \pitchfork{ }_{1}^{1} U D S_{0}+R B_{1}^{1}+(1 i p){ }^{i} \pitchfork O U D S_{0}+R^{2} B_{0}{ }^{\phi} . \quad\left(U D S_{0} i K\right)^{+} \\
& p^{i} \phi_{1}^{2} U D S_{0}+R B_{1}^{2}+(1 ; p){ }^{i} \phi D_{0}^{2} S_{0}+R^{2} B_{0} \phi . \quad\left(U D S_{0} i K\right)^{+} \\
& p^{i} \phi{ }_{1}^{2} D^{2} S_{0}+R B_{1}^{2}+(1 ; p){ }^{i} \pitchfork D^{2} S_{0}+R^{2} B_{0}{ }^{\phi} . \quad{ }^{i} D^{2} S_{0} i K^{\phi_{+}}
\end{aligned}
$$


positive expected payo ${ }^{\circledR}$ at time $t=1$;

$$
\begin{aligned}
& { }^{i}{ }^{1}{ }_{1}^{1} U^{2} S_{0}+R B_{1}^{1}{ }_{1}^{\Phi} . \quad{ }^{i} U^{2} S_{0} i K^{\Phi_{+}} \\
& { }^{i}{ }_{1}^{1} U D_{0}+R B_{1}^{1} \phi \quad\left(U D S_{0} i K\right)^{+} \\
& i{ }_{1}^{2} U S_{0}+R B_{1}^{2} \phi .\left(U^{2} S_{0} K\right)^{+} \\
& \mathrm{i}^{\mathrm{i}}{ }_{1}^{2} \mathrm{D}^{2} \mathrm{~S}_{0}+\mathrm{RB}_{1}^{2}{ }^{\Phi} \cdot \mathrm{i}^{2} \mathrm{D}^{2} \mathrm{~S}_{0} \mathrm{~K}^{\Phi_{+}}
\end{aligned}
$$

and self-" nancing,

$$
\begin{array}{ll}
\$_{1}^{1} U S_{0}+B_{1}^{1}, & \$ 0 U S_{0}+R B_{0} \\
\uparrow_{1}^{2} D S_{0}+B_{1}^{2}, & \$ D_{0} D S_{0}+R B_{0}:
\end{array}
$$

The Lagrangean of the problem is

$$
\begin{aligned}
& L=\phi_{0} S_{0}+h_{\mathrm{B}_{0}}+
\end{aligned}
$$

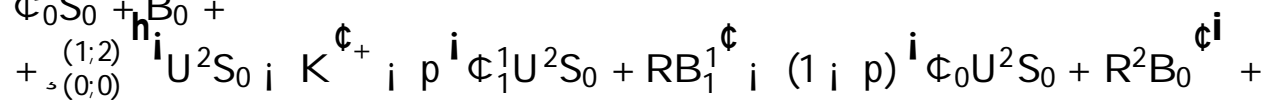

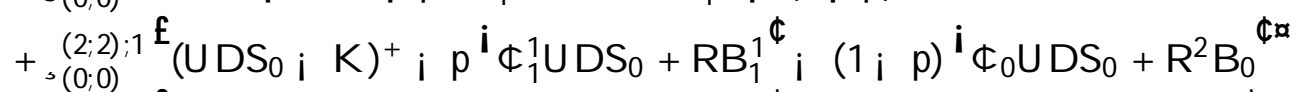

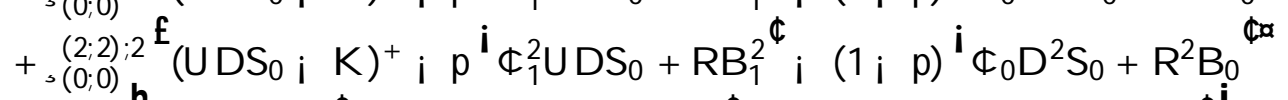

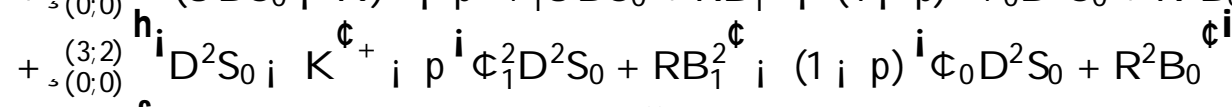

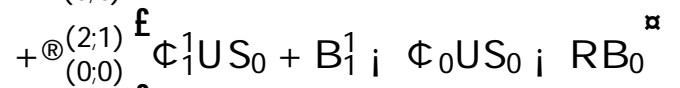

$$
\begin{aligned}
& +\otimes_{0 ; 0)}^{(1 ; 1)} f_{\phi_{1}} D S_{0}+B_{1}^{2} i \quad\left(\phi{ }_{0} D S_{0}+R B_{0}\right)^{\alpha} \\
& +,(1 ; 2) \mathrm{i}^{2} \mathrm{U}^{2} \mathrm{~S}_{0} \mathrm{i} \mathrm{K}^{\Phi_{+}} \mathrm{i}^{\mathrm{i}} \phi_{1}^{1} \mathrm{U}^{2} \mathrm{~S}_{0}+\mathrm{RB}_{1}^{1}{ }^{\mathrm{C}} \\
& +\underset{+(2 ; i)}{(2 ; 2)} f_{\left(U D S_{0} i K\right)^{+} i} i_{\phi}{ }_{1}^{1} U D S_{0}+R B_{1}^{1}{ }^{\phi \alpha}
\end{aligned}
$$

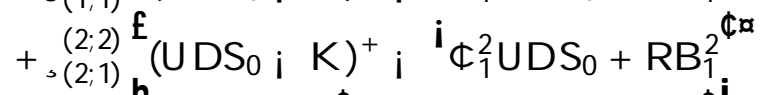

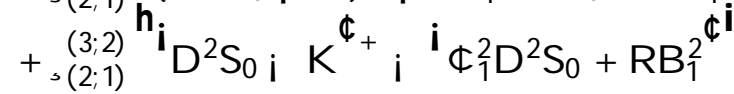

The constraints that are binding depend on the value of the parameters. First, if $R^{2} i$ UD $<0$ and $p>\frac{U D ; R^{2}}{U D_{i} R^{2}+R(U i D)}$ or $R^{2} i U D>0$ and $p>$ $\frac{R^{2} i U D}{R^{2} i U D+R\left(U_{i} D\right)}$ the optimal solution of the dual problem is characterized by

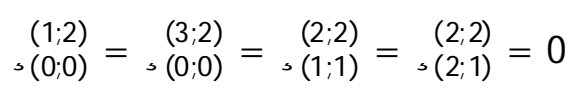


and

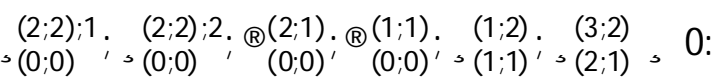

Second, if $R^{2} i \quad U D<0$ and $p<\frac{U D_{i} R^{2}}{U D_{i} R^{2}+R\left(U_{i} D\right)}$ the optimal solution of the dual problem is characterized by

$$
\stackrel{(1 ; 2)}{,(0 ; 0)}=\stackrel{(2 ; 2) ; 1}{,(0 ; 0)}=\stackrel{(1 ; 2)}{,(1 ; 1)}=\stackrel{(2 ; 2)}{,(1 ; 1)}=\mathbb{R}(1 ; 1)=0
$$

and

$$
\begin{array}{ll}
(2 ; 2) ; 2 & (3 ; 2) ;(2 ; 1) ;(2 ; 2) ;(3 ; 2), 0:
\end{array}
$$

Finally, if $R^{2} i \quad U D>0$ and $p<\frac{R^{2} i U D}{R^{2} i U D+R\left(U_{i} D\right)}$ the optimal solution of the dual problem is characterized by

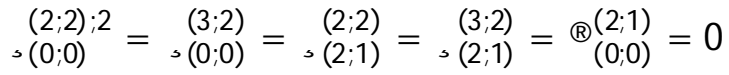

and

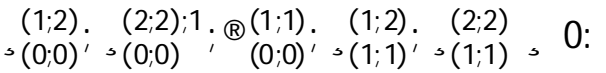

Hence, after some algebra the lower bound can be written as:

$$
C_{l}=q^{1}{ }^{f} U^{2} S_{0} \text { i } K^{x_{+}}+q^{2}\left[U_{D S} \text { i } K\right]^{+}+q^{3}{ }^{f} S^{2} S_{0} \text { i } K^{x_{+}}
$$

with

$$
\begin{aligned}
& q^{1}=\frac{(R ; \quad D)^{2}{ }^{i} U D i R^{2^{\Phi}}+p(U ; \quad R)(R ; \quad D)^{i} D^{2} i R^{2^{\Phi}}}{R^{2}(U ; R)^{2}\left(D^{2} ; \quad U D\right)+(R ; \quad D)^{2}\left(U D ; D^{2}\right)+p(U ; R)(R ; \quad D)\left(D^{2} ; \quad U^{2}\right)} \\
& q^{2}=\frac{(U ; R)^{2}{ }^{i} D^{2} i R^{2}+(R ; D)^{2}{ }^{4} R^{2} i U^{2}}{R^{2}(U ; R)^{2}\left(D^{2} i U D\right)+(R ; D)^{2}\left(U D ; D^{2}\right)+p(U ; R)(R ; D)\left(D^{2} ; U^{2}\right)} \\
& q^{3}=\frac{h \quad(U ; R)^{2} R^{2} i U D^{\Phi}+p(U ; R)(R ; D){ }^{i} R^{2} i U^{2}}{R^{2}(U ; R)^{2}\left(D^{2} i U D\right)+(R ; D)^{2}\left(U D ; D^{2}\right)+p(U ; R)(R ; D)\left(D^{2} i U^{2}\right)} \\
& \text { if } R^{2} i U D<0 \text { and } p>\frac{U D_{i} R^{2}}{U D_{i} R^{2}+R\left(U_{i} D\right)} \text { or } R^{2} i U D>0 \text { and } p> \\
& \frac{R^{2} i U D}{R^{2} i U D+R\left(U_{i} D\right)} \text {, } \\
& C_{1}=\frac{1}{R^{2}} \frac{R^{2} i D^{2}}{D(U ; D)}\left[U_{D} S_{0} i K\right]^{+}+\frac{U D i R^{2}}{D(U ; D)} f^{2} S_{0} i K^{a_{+}}
\end{aligned}
$$


if $R^{2} i U D<0$ and $p<\frac{U D_{i} R^{2}}{U D_{i} R^{2}+R\left(U_{i} D\right)}$ and

$$
C_{1}=\frac{1}{R^{2}} \frac{R^{2} i U D}{U(U i D)}{ }^{f} U^{2} S_{0} i K^{b_{+}}+\frac{U^{2} i R^{2}}{U(U ; \quad D)}\left[U D S_{0} i K\right]^{+}
$$

if $R^{2} i U D>0$ and $p<\frac{R^{2} i U D}{R^{2}{ }_{i} U D+R\left(U_{i} D\right)}$ :

\section{B.3 Example on P roperty 3}

Example 22 (for $T=2$ ) When $p$ ! 0 , which means that the market is completely illiquidity, the values of the lower bound are given by (27) or (28) depending on $R^{2} i$ UD 70 . These values coincide with the ones presented in (2) for $T=2$. On the other hand, when $p$ ! 1 , which means that there is no illiquidity, the values of $q_{1} ; q_{2}$ and $q_{3}$ presented in (26) tend to

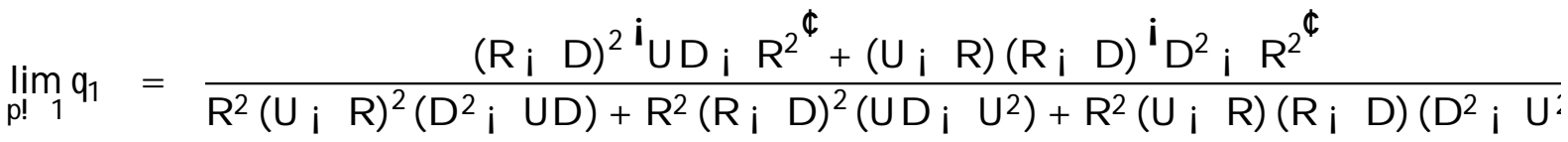

$$
\begin{aligned}
& =\frac{(R ; D)^{2}}{R^{2}\left(U_{i} D\right)^{2}} \\
& \lim _{p ! 1} q_{D}=\frac{(U ; R)^{2} D^{2} i R^{2^{\Phi}}+(R ; D)^{2 i} R^{2} i U^{2^{\Phi}}}{R^{2}(U ; R)^{2}\left(D^{2} i U D\right)+R^{2}(R ; D)^{2}\left(U D i D^{2}\right)+R^{2}(U ; R)(R ; \quad D)\left(D^{2} i U\right.}
\end{aligned}
$$$$
=2 \frac{(R ; D)(U i R)}{R^{2}(U i D)^{2}}
$$$$
\lim _{p ! 1} \phi_{B}=\frac{(U ; R)^{2}{ }^{i} R^{2} i U D^{\Phi}+(U ; R)(R ; D){ }^{i} R^{2} i U^{2}}{R^{2}(U ; R)^{2}\left(D^{2} i U D\right)+R^{2}(R ; D)^{2}\left(U D ; D^{2}\right)+R^{2}(U ; R)(R ; \quad D)\left(D^{2} ; U\right.}
$$$$
\frac{(U ; R)^{2}}{R^{2}(U ; D)^{2}}
$$

then,

$$
\begin{aligned}
& \lim _{p ! 0} C_{l}=\frac{\left(R_{i} D\right)^{2}}{R^{2}(U ; D)^{2}}{ }^{f} S_{0} i K^{a_{+}}+2 \frac{(R ; D)(U ; R)}{R^{2}(U ; D)^{2}}\left[U D S_{0} i K\right]^{+} \\
& +\frac{(U ; R)^{2}}{R^{2}(U ; D)^{2}}{ }^{f} S_{0} i K^{b_{+}}
\end{aligned}
$$

which coincides with $C_{1}$; presented in equation (1). 